University of Louisville

ThinkIR: The University of Louisville's Institutional Repository

Electronic Theses and Dissertations

8-2011

\title{
Evaluation of methods for analyzing gene-gene interaction data for survival outcomes.
}

Jie Zhang

University of Louisville

Follow this and additional works at: https://ir.library.louisville.edu/etd

\section{Recommended Citation}

Zhang, Jie, "Evaluation of methods for analyzing gene-gene interaction data for survival outcomes." (2011). Electronic Theses and Dissertations. Paper 1640.

https://doi.org/10.18297/etd/1640

This Master's Thesis is brought to you for free and open access by ThinkIR: The University of Louisville's Institutional Repository. It has been accepted for inclusion in Electronic Theses and Dissertations by an authorized administrator of ThinkIR: The University of Louisville's Institutional Repository. This title appears here courtesy of the author, who has retained all other copyrights. For more information, please contact thinkir@louisville.edu. 


\title{
EVALUATION OF METHODS FOR ANALYZING GENE-GENE INTERACTION DATA FOR SURVIVAL OUTCOMES
}

By

Jie Zhang

B.E., China Pharmaceutical University, 2007

M.S. program, China Pharmaceutical University, 2009

\author{
A Thesis \\ Submitted to the Faculty of the \\ Graduate School of the University of Louisville \\ in Partial Fulfillment of the Requirements \\ for the Degree of \\ Master of Science \\ Department of Bioinformatics and Biostatistics \\ School of Public Health and Information Sciences \\ University of Louisville \\ Louisville, Kentucky
}

August 2011 

EVALUATION OF METHODS FOR ANALYZING GENE-GENE INTERACTION DATA FOR SURVIVAL OUTCOMES

\author{
By \\ Jie Zhang \\ B.E., China Pharmaceutical University, 2007 \\ M.S. program, China Pharmaceutical University, 2009
}

A Thesis Approved on

May $12^{\text {th }}, 2011$

by the following Thesis Committee:

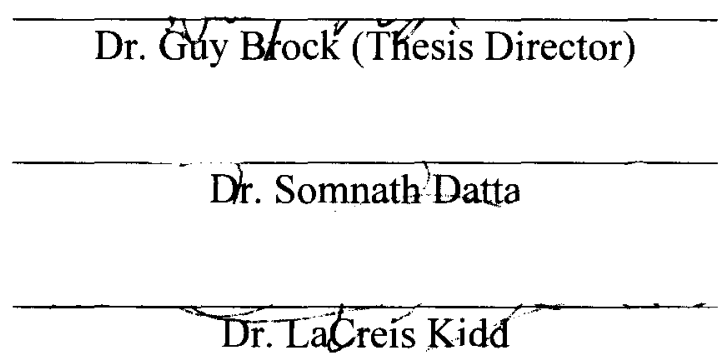




\section{DEDICATION}

This thesis is dedicated to my parents

Mr. Chuansheng Zhang

and

Mrs. Xueping Wang

who have given me invaluable educational opportunities. 


\section{ACKNOWLEDGMENTS}

I would like to thank all the people who made this work possible. First, I would like to thank my advisor, Dr. Guy Brock, for his moral and financial support during my course work at University of Louisville. I thank Dr. Brock for being very patient and supportive in guiding me throughout this project. I would also like to thank Dr. LaCreis Kidd for giving me an opportunity to work on the related project for 9 months. I appreciate the guidance and financial support I received from her. Finally, I would like to convey my sincere thanks to Dr. Somnath Datta, for his presence and comments. 


\section{ABSTRACT \\ EVALUATION OF METHODS FOR ANALYZING GENE-GENE INTERACTION DATA FOR SURVIVAL OUTCOMES}

Jie Zhang

May 12, 2011

In recent years, a number of computational and statistical problems for identifying SNP-SNP interactions in high dimensional survival data have been studied, and several data mining approaches have been proposed. However, the relative performance of these methods to detect SNP-SNP interactions has not been thoroughly investigated.

In this study, we directly compared the performance of the four techniques to detect gene-gene interactions in a recently conducted study of genetic polymorphisms associated with breast cancer survival and recurrence. Four methods were evaluated for their ability to detect SNP-SNP interactions: Survival Multifactor Dimensionality Reduction, Cox regression with $\mathrm{L}_{1}$ (Lasso) and $\mathrm{L}_{1}-\mathrm{L}_{2}$ (Elastic Net) penalties, and Random Survival Forest (RSF). Methods were contrasted on the basis of which SNPs they selected.

The results of this study demonstrate how the methods perform in detecting gene-gene interactions for survival data, and are useful in informing researchers about choosing an analysis tool for their own real data applications. 


\section{TABLE OF CONTENTS}

PAGE

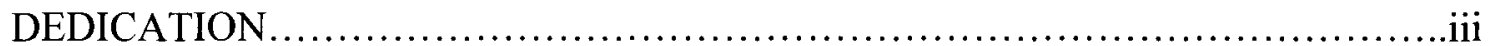

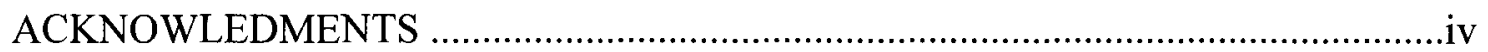

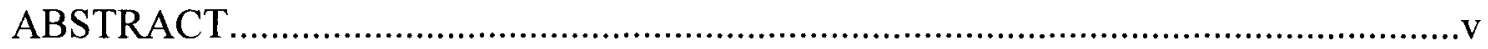

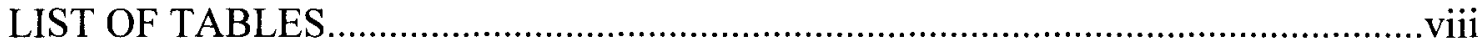

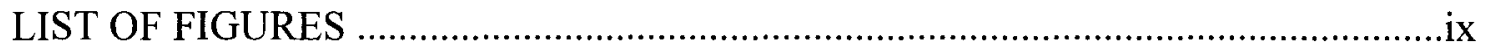

\section{CHAPTER}

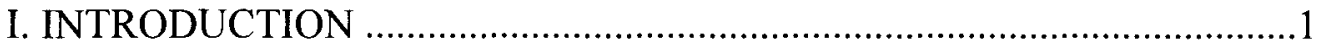

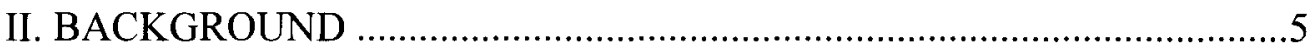

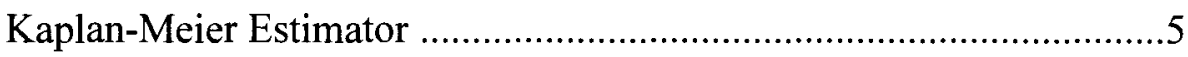

Log-rank test..................................................................................

Cox proportional hazard model ........................................................6

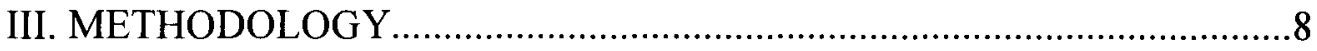

Penalized likelihood methods.....................................

$\mathrm{L}_{1}$-penalized cox regression.............................. 9

$\mathrm{L}_{2}$-penalized cox regression ...............................

Elastic Net...........................................10

Cross-validation and optimization............................10

Survival Multifactor Dimensionality Reduction ......................10

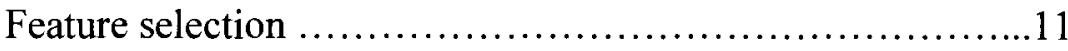

Model validation......................................... 12

Random Survival Forest ..........................................13

The algorithm............................................

Splitting rules........................................... 


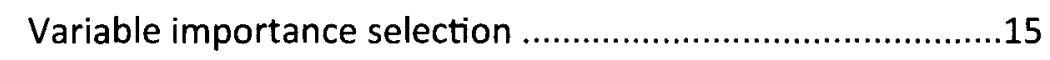

Minimal depth selection ................................................15

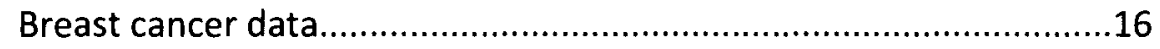

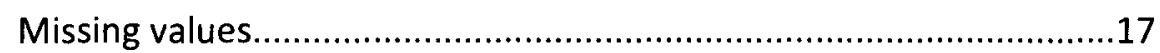

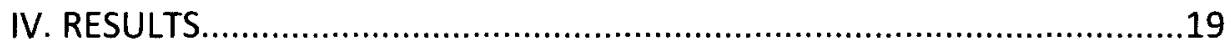

Penalized Cox regression models.................................................19

$\mathrm{L}_{1}$-penalized cox regression..........................19

Elastic Net .......................................21

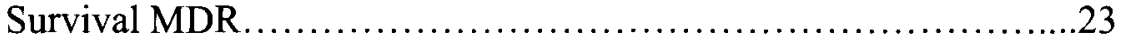

Random Survival Forest ...................................25

Comparison of results .................................... 26

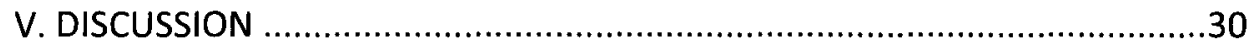

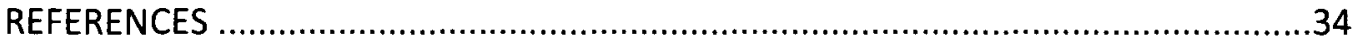

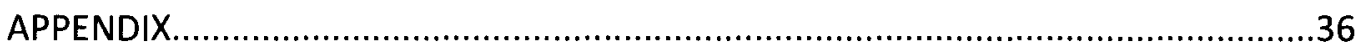

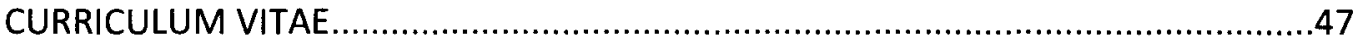




\section{LIST OF TABLES}

TABLE

PAGE

1. Selected SNPs in the angiogenesis pathway and their nucleotide change........17

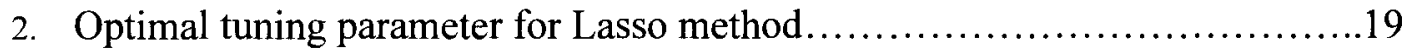

3. Selected models based on Lasso method .................................20

4. Optimal tuning parameter for Elastic Net method .........................22

5. Selected models based on Elastic Net method .......................................22

6. Selected models based on Survival MDR method ........................24

7. Selected models based on Random Survival Forest method ...................25

8. Comparison table of significant variables selected by different methods based on

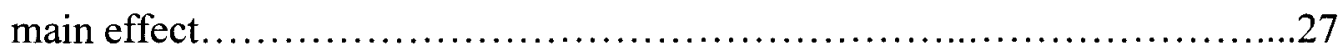

9. Comparison table of significant variables selected by different methods based on

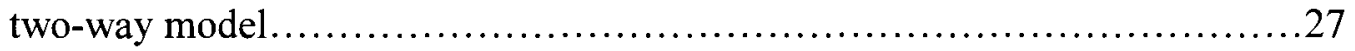

10. Comparison table of significant variables selected by different methods based on three-way model....................................................28 


\section{LIST OF FIGURES}

FIGURE

PAGE

1. Survival MDR attribution construction

2. Ten-fold cross-validation.............................................. 12

3. Kaplan-Meier survival curve for high and low risk groups based on Lasso two-way and three-way models.

4. Kaplan-Meier survival curve for high and low risk groups based on Elastic Net onetwo-, and three-way models.

5. Kaplan-Meier survival curve for high and low risk groups based on Survival MDR one-, two-, and three-way models.

6. Variables clustered by second-order minimal depth.......................26

7. Kaplan-Meier survival curve for high and low risk groups based on Lasso, Elastic Net, and Survival MDR. .28

8. Kaplan-Meier survival curve for high and low risk groups based on VIMP and minimal depth one-way models. 


\section{CHAPTER I}

\section{INTRODUCTION}

Technological advances are rapidly improving geneticist's ability to detect genetic factors, which influence individual susceptibility to common human diseases. Research has shown that genes don't function alone; rather, they constantly interact with one another. Given the complexity and robustness of biological networks such diseases are unlikely to be the result of single genetic variants, but instead arise from the joint interaction of two or more variants acting together.

When we think about the whole biological networks, it is important to consider epistasis, which refers to the situation where interacting genes, as opposed to a single gene, influence a trait. The methods to detect and characterize epistasis are critical to understanding complex diseases, such as Alzheimer's disease, diabetes, cardiovascular disease, and cancer.

One traditional approach to modeling the relationship between discrete predictors such as genotypes and discrete clinical outcomes is logistic regression modeling (Lecessie and Vanhouwelingen, 1992). Logistic regression is a parametric statistical approach for relating one or more independent or explanatory variables (e.g. disease status) that follows a binomial distribution. However, detecting and characterizing epistatic interactions in datasets containing large numbers of SNPs is challenging. The 
number of possible interaction terms grows exponentially as each additional main effect is included in the logistic regression model. Thus, logistic regression is limited in its ability to deal with interaction data.

In response to this limitation, the multifactor dimensionality reduction (MDR) (Ritchie et al., 2001) has been developed as a method for reducing the dimensionality of multi-locus information, to improve the identification of polymorphism combinations associated with disease risk. This approach is nonparametric, is free of a specified genetic model, and is directly applicable to the analysis of case-control and discordant sib-pair study designs. Both empirical and theoretical studies suggest that MDR has excellent power for identifying high-order gene-gene interactions and is a promising new approach for overcoming some of the limitations of logistic regression.

Another machine learning method that has attracted considerable interest is Random Forests (RF) (Breiman, 2001). It is a technique that builds a forest of classification trees. Each single tree is grown from a bootstrap sample of the data, and the final outcome predictions are determined by all trees in the forest. There are several advantages of the RF method. It can handle a large number of input variables. It is fairly robust in dealing with genetic data in the presence of high amounts of missing data.

Recently, many researchers have been interested in identifying gene-gene interactions in high dimensional survival data. Thus, the above methods have been extended to survival analysis. Survival analysis involves the modeling of time to event data; in this context, death or failure is considered an "event". Survival analysis attempts to answer questions such as "What is the fraction of a population that will survive past a certain time?". To model survival data, there are a few basic tools such as the Kaplan- 
Meier estimate of the survival function (Kaplan and Meier, 1958), the log-rank test for differences between survival functions (Breslow, 1975), and the Cox proportional hazards model for modeling the effect of multiple covariates on the hazard rate (Cox, 1972). Although numerous computational and statistical methods for identifying SNPSNP interactions in case-control studies have been studied extensively, the analysis of SNP-SNP interactions associated with survival data is relatively uninvestigated in the literature.

In the case that survival outcomes associated with genomic data, we have two statistical objectives. The first is to identify which of the features (e.g. genes or SNPs) in the genomic data is individually most associated with the survival outcome, and the second is build a model which can accurately predict survival times. Several methods have recently been proposed in the literatures that address the two objectives mentioned above. One approach is to use penalized Cox regression (Benner et al., 2010) in such situations. This method determines a subset of covariates that are the most important ones for predicting the survival outcome by shrinking unimportant regression coefficients to zero using penalties on the magnitude of the regression coefficients. Another parametric approach is the accelerated failure time model, which has been applied in conjunction with partial least squares and the least absolute shrinkage and selection operator (Lasso) to predict patient survival times from high-dimensional microarray data (Datta et al., 2007). Other, nonparametric approaches include the Survival Multifactor Dimensionality Reduction algorithm (Survival-MDR) (Gui et al., 2011), and Random Survival Forests (RSF) (Ishwaran et al., 2008). Survival-MDR uses a multifactor dimensionality reduction algorithm that reduces the $\mathrm{N}$-dimensional space for $\mathrm{N}$-way interactions between SNPs to 
one dimension with two levels ("low risk" and "high risk"). Random Survival Forest is a technique that builds a forest of classification trees, wherein each single tree is grown from a bootstrap sample of the data, and predictions of patients with unknown survival times are determined by aggregation of all the trees in the forest.

In the current thesis, we evaluate the use of penalized Cox regression models, Survival-MDR, and RSF for detecting gene-gene interactions associated with breast cancer $(\mathrm{BrCa})$ survival and recurrence. Each method is used to identify important single nucleotide polymorphisms (SNPs) which contribute to one-, two-, and three-way interaction models for BrCa survival and recurrence. The rest of this thesis is organized as follows. In chapter 2, we describe some background methodology for survival analysis including the Kaplan-Meier estimator, the log-rank test, and the Cox proportional hazards model. Chapter 3 describes two penalized Cox regression models, Survival-MDR, and $\mathrm{RSF}$, and also describes the breast cancer dataset used in this research and the data management technique. Chapter 4 summarizes the findings from using the above four methods. Chapter 5 gives some concluding remarks and discusses future research. 


\section{CHAPTER II}

\section{BACKGROUND}

\subsection{Kaplan-Meier Estimator}

The Kaplan-Meier estimator is a nonparametric estimator of the survival function for right-censored data. It is also known as product-limit estimator. Let $S(t)$ be the probability that an item from a given population will have a lifetime exceeding $t$. The Kaplan-Meier estimator is the nonparametric maximum likelihood estimate of $S(t)$. It is a product of the form

$$
\hat{S}(t)=\prod_{t_{i} \leq t}\left(\frac{n_{i}-d_{i}}{n_{i}}\right)
$$

where $t$ is the $i^{\text {th }}$ distinct observed failure time, $i=1,2, \ldots, D, D$ is the number of distinct observed failure times, $d_{i}$ is the number of individuals who experience the event of interest at time $t_{i}$, and $n_{i}$ is the number of individuals at risk just before time $t_{i}$.

\subsection{Log-rank test}

The log-rank test statistic is widely used to compare two independent survival functions in the presence of censored observations. The test statistic is based on a normalized sum of the differences between the observed failures and the expected 
failures in either of the treatment groups, and has an asymptotic chi-square distribution with one degree of freedom.

Let $j=1, \ldots, J$ be the set of distinct times of observed events in either group. Let $N_{1 j}$ and $N_{2 j}$ be the number of subjects which have not yet had an event or been censored at time jfor groups 1 and 2, respectively, and define $N_{j}=N_{1 j}+N_{2 j}$. For each time $j$, let $O_{1 j}$ and $O_{2 j}$ be the observed number of events and $O_{j}=O_{1 j}+O_{2 j}$. Under the null hypothesis of equal survival functions for subjects in groups 1 and 2, the expected number of failures at timetis expressed as

$$
E_{1 j}=O_{j} \frac{N_{1 j}}{N_{j}}
$$

The $\log$ rank statistic $Z^{2}$ compares each $O_{1 j}$ to its expectation $E_{1 j}$ under the null hypothesis, and is defined as

$$
Z^{2}=\frac{\left(\sum_{j=1}^{J}\left(O_{1 j}-E_{1 j}\right)\right)^{2}}{\sum_{j=1}^{J}\left(\frac{O_{j}\left(N_{1 j} / N_{j}\right)\left(1-N_{1 j} / N_{j}\right)\left(N_{j}-O_{j}\right)}{N_{j}-1}\right)}
$$

The statistic $Z^{2}$ has an asymptotic chi-squared distribution with one degree of freedom, under the assumption of the null hypothesis.

\subsection{Cox proportional hazard model}

The Cox proportional hazards model is commonly used to model survival data. It is non-parametric in that the baseline hazard function can take an arbitrary form. The model is as follows: 


$$
\lambda\left(t \mid x_{i}\right)=\lambda_{0}(t) \exp \left(\sum_{j=1}^{p} x_{i j} \beta_{j}\right)
$$

where $x$ are covariates, $\lambda\left(t \mid x_{i}\right)$ is the hazard at time $t$ for the $i$ th observation, $\lambda_{0}(t)$ is the unspecified baseline hazard function, and $\beta$ is a vector of regression coefficients. The partial likelihood is given by

$$
L(\beta)=\prod_{i: C_{i}=1} \frac{\exp \left(x_{i} \beta\right)}{\sum_{j: Y_{j} \geq Y_{i}} \exp \left(x_{j} \beta\right)}
$$

where $Y_{i}$ denotes the observed time (either censoring time or event time) for subject $i$, and $C_{i}$ is the indicator that the time corresponds to an event $\left(C_{i}=1\right.$ if the event occurred and $C_{i}=0$ if the time is a censoring time ). The log partial likelihood is given by

$$
l(\beta)=\sum_{i: C_{i}=1}\left(\beta x_{i}-\log \sum_{j: Y_{j} \geq Y_{i}} \exp \left(x_{j} \beta\right)\right)
$$

Parameter estimates $\hat{\beta}$ in the Cox proportional hazard model are obtained by maximizing the partial likelihood. 


\section{CHAPTER III}

\section{METHODOLOGY}

\subsection{Penalized likelihood methods}

Penalized likelihood methods(Benner et al., 2010) can be used for determining a subset of covariates that are the most important for predicting survival times, when we fit models for survival data on the basis of the Cox proportional hazards model. The penalized log partial likelihood is given by

$$
l(\beta)-\sum_{j=1}^{p} p_{\lambda}\left(\left|\beta_{j}\right|\right),
$$

where $l(\beta)$ denotes the log partial likelihood, $p$ is the dimension of the parameter $\beta, j$ is the index with parameter estimates unequal to zero, and $p_{\lambda}\left(\left|\beta_{j}\right|\right)$ is the penalty function with a tuning parameter $\lambda$. The penalty term on the coefficients will shrink them towards zero, thus reducing the dimensionality of the problem and increasing the stability of the solution.

We describe $k$-way $(k=1,2,3)$ interaction models that capture the relationship between $k$ genes and the survival outcome. For the one way model, the penalized Cox regression model will include just main effects. For the two-way model, both main and two-way interaction effects are considered. For the three-way model, main effects, two-, and three-way interaction effects are all used to fit the model. 
In order to determine the most important SNPs for the penalized Cox regression models, we rank the coefficients by their absolute value. The larger the absolute value, the more important the SNP.

\subsection{1 $\mathrm{L}_{1}$-penalized cox regression}

Based on the penalized likelihood methods, Tibshirani (Tibshirani, 1997) proposed to use the $\mathrm{L}_{1}$-penalized Cox regression model and described a technique, called the Lasso for "Least absolute shrinkage and selection operator", for parameter estimation. The $\mathrm{L}_{1}$-penalized Cox model has the following penalty,

$$
p_{\lambda}\left(\left|\beta_{j}\right|\right)=\lambda\left|\beta_{j}\right|, j=1, \ldots, p
$$

By penalizing the coefficients associated with each of the covariates during model fitting, unimportant coefficient will be shrunk towards zero. Thus, the Lasso can be considered as a version of automated variable selection, particularly useful for high-dimensional data.

\subsection{2 $\mathrm{L}_{2}$-penalized cox regression}

$\mathrm{L}_{2}$-penalized Cox regression with the ridge penalty

$$
p_{\lambda}\left(\left|\beta_{j}\right|\right)=\lambda \beta_{j}^{2}, j=1, \ldots, p
$$

was introduced by Verweij and Van Houwelingen (Verweij and Van Houwelingen, 1994). The ridge regression shrinks the regression coefficients in a manner similar to lasso regression, but penalizes the square values instead of the absolute values. Regression coefficients are not shrunk completely to zero, and thus ridge regression is not a variable selection algorithm. Rather, the procedure is particularly useful for stabilizing the beta coefficients when the set of regression variables are highly correlated. 


\subsubsection{Elastic Net}

Elastic net, which employs a combination of the $L_{1^{-}}$and $L_{2}$-penalties, was introduced by Zou and Hastie (Zou and Hastie, 2005)

$$
p_{\lambda_{1}, \lambda_{2}}\left(\left|\beta_{j}\right|\right)=\lambda_{1}\left|\beta_{j}\right|+\lambda_{2} \beta_{j}^{2}, j=1, \ldots, p .
$$

The Elastic Net performs variable selection similarly to the Lasso. But the additional $\mathrm{L}_{2}$ penalty distributes the weight to more variables, such that the Elastic Net tends to select more variables than the Lasso.

\subsubsection{Cross-validation and optimization}

The penalized regression models use cross-validation of the log partial likelihood to assess its predictive ability and determine the optimal values of the tuning parameters $\lambda_{1}$ and $\lambda_{2}$. The tuning parameters are chosen by maximizing the $k$-fold cross-validated log partial likelihood. In our study, we used 10-fold cross-validation. The tuning parameter $\lambda_{1}$ was chosen by maximizing 10 fold cross-validated log likelihood, while the tuning parameter $\lambda_{2}$ for Elastic Net was chosen from the set $\{0.0001,0.001,0.01,0.1,1$, $10\}$

\subsection{Survival Multifactor Dimensionality Reduction (Survival MDR)}

Survival MDR is a nonparametric and genetic model-free approach that reduces the dimensionality of the data when analyzing gene-gene interactions. It is an extension of the MDR approach developed for case-control data, and is specifically designed to characterize gene-gene interactions in the presence of right-censored data. The goal of Survival MDR is to change the representation of the data using a constructive induction 
algorithm, to make interactions easier to detect. The procedure will be used for both feature selection and model validation as described next.

\subsubsection{Feature selection}

The Survival MDR procedure for feature selection is illustrated in Figure 1. For determining an $\mathrm{N}$-way interaction amongst a pool of SNPs, the algorithm proceeds through the following three steps for all possible $N$-way combinations of SNPs.
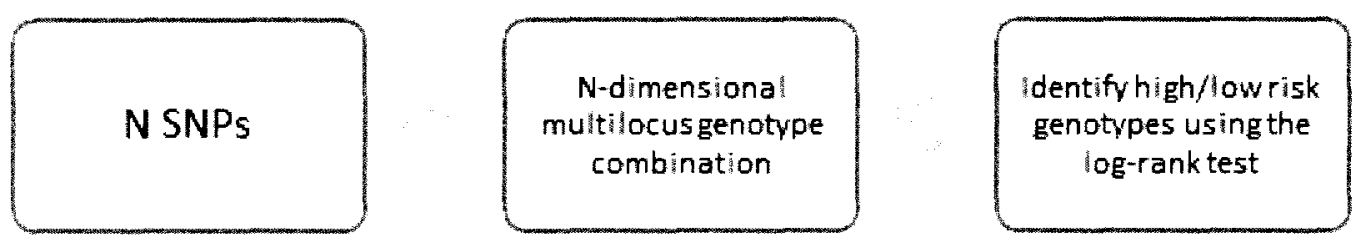

Fig. 1 Survival MDR attribution construction

First, $N$ SNPs are selected from the pool of all SNPs. In the second step, the logrank test statistics comparing the survival time distributions with and without each of the multilocus genotype combinations in the $N$-way interaction table are calculated. In the third step, each multifactor cell in the $N$-dimensional space is classified as either "high risk," if the log-rank test statistic is positive, or as "low risk," if the log-rank statistic is negative. Once all the genotype are labeled as either "high risk" or "low risk", the dimensionality of multi-locus genotype information is reduced to one dimension with two levels (“low risk" and "high risk"). 


\subsubsection{Model validation}

Survival MDR uses the log rank test to compare the survival time distributions between high and low risk groups and uses $Z^{2}$ as the score to choose the best model amongst all possible $N$-way combinations of SNPs. A ten-fold cross-validation procedure is used to determine the best overall model, involving three steps.

In the first step, the whole dataset is randomly divided into ten equal parts. Ninetenths of the data are used as the training set, and the remaining one-tenth of the data are set aside as an independent testing set. Then $Z^{2}$ statistic is computed for each $N$-way interaction model in the training set.

In the second step, an attribute is created for the independent testing set using the $N$ SNPs that have the best $Z^{2}$ score. To reduce the possibility of poor estimates of the predictive ability due to chance divisions of the data set, the entire procedure is performed 10 times so that each sample is included in the testing set at least once.

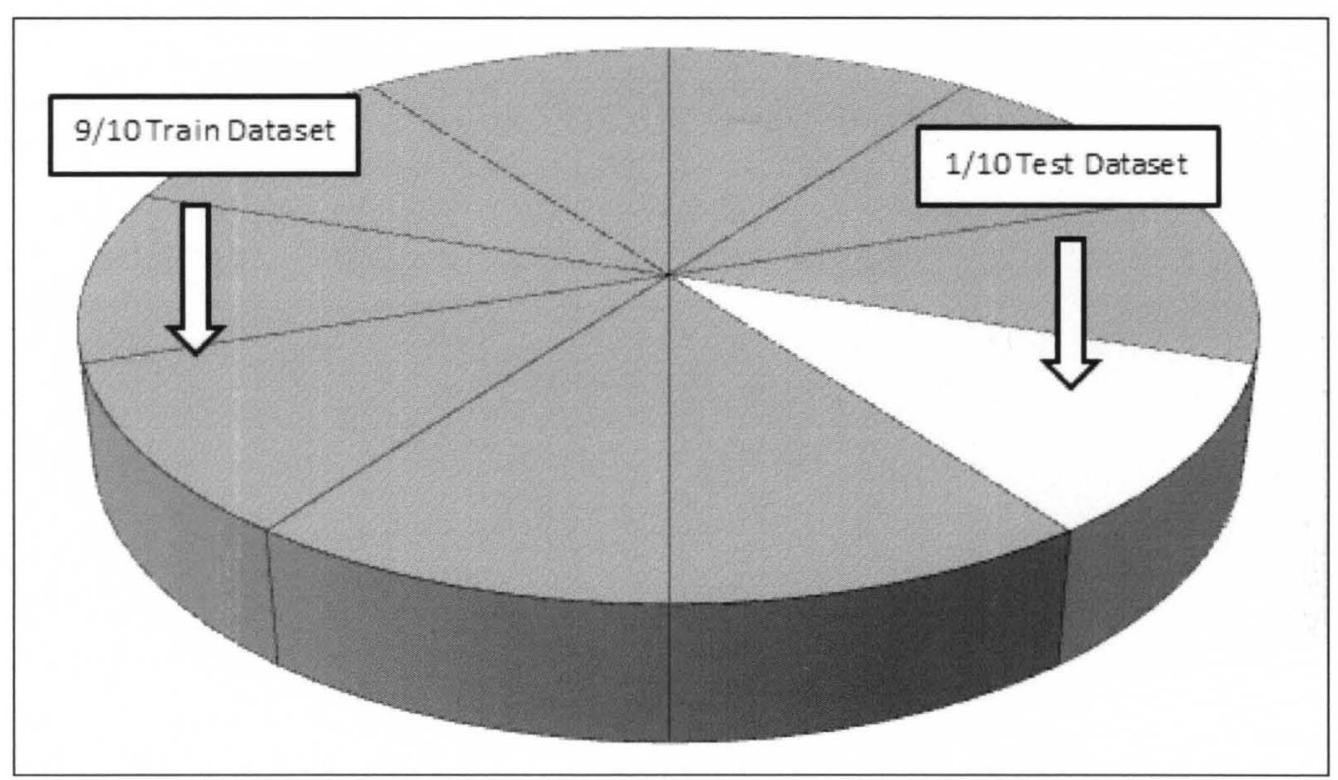

Fig 2.Ten-fold cross-validation. 
In the third step, for each 10 -fold cross-validation, the number of times the same best set of loci/factors was identified across the 10 data subsets is recorded. The crossvalidation consistency $(\mathrm{CVC})$ is a measure of the number of times a particular set of loci and/or factors is identified across the cross-validation subsets. There are 10 possible values of the CVC ranging from 1 to 10 , where 10 indicate strong evidence in favor of a multifactor association. The best Survival MDR model was selected as that with the maximum testing accuracy averaged over the 10 -fold cross validation, and also has the maximum cross-validation consistency (CVC) as described previously. For all of the selected models, the CVC ranged from 8 to 10 are considered as a highly predictive model.

\subsection{Random Survival Forests}

Random Survival Forests(RSF) are an ensemble tree method for the analysis of right censored survival data. Standard analyses often rely on restrictive assumptions such as proportional hazards. This property is especially helpful in survival analysis, and makes RSFs highly data adaptive. The procedure only requires three parameters to be set (the number of randomly selected predictors, the number of trees grown, and the splitting rule used), thus making it quite user-friendly.

\subsubsection{The Algorithm}

Each tree in the forest is constructed as follows: 
In the first step, draw $B$ bootstrap samples, with replacement, from the entire data set. $B$ is the total number of trees in the forest. Each bootstrap sample excludes on average $37 \%$ of the data, called the out-of bag data (OOB data).

In the second step, a survival tree grows for each bootstrap sample. At each node in the tree, randomly select $m$ candidate variables for splitting on. The absolute magnitude of $m$ is a function of the number of variables in the data set and remains constant throughout the forest building process.

In the third step, split on a variable using one of several survival splitting rules. A node is split on that variable which maximizes the survival differences between daughter nodes.

Computation proceeds by iterating the second and third steps until the tree is fully grown (no pruning).

\subsubsection{Splitting rules}

Four different splitting rules are available for use with RSF(Ishwaran et al., 2008).The log-rank splitting rule, the default splitting rule, splits nodes by maximizing the log-rank test statistic. In this study, we the analysis under this splitting rule since is a well-established concept, having been shown to be robust in both proportional and nonproportional hazards settings. Variants on this procedure include the log-rank score rule, which splits nodes on the basis of a standardized log-rank statistic, and the random logrank splitting rule, which is a fast approximation to the log-rank splitting rule. As an alternative to the log-rank splitting rules, node splitting can be based instead on the 
conservation-of-events principle. This node splitting rule may help to reduce the potential bias in the other splitting rules to favor continuous predictors and uneven splits.

\subsubsection{Variable importance (VIMP) selection}

Variables can be selected by filtering on the basis of their variable importance (VIMP). For each bootstrap sample, $37 \%$ of the data are excluded on average. Thus, for a given tree, certain individuals will have been left out of the training data. Prediction error and variable importance is estimated from these 'out-of-bag' (OOB) data.

The OOB data is used to estimate the importance of particular variables by randomly permuting the values of that variable and testing whether these permutations adversely affect the predictive ability of trees in classifying the OOB data. If randomly permuting values of a particular variable does not affect the predictive ability of trees on OOB samples, that variable is assigned a low importance score. Conversely, if randomly permuting the values of a particular variabledrastically impairs the ability of trees to correctly predict the survival time of the OOB samples, then the importance score of that variable will be high.

\subsubsection{Minimal depth selection}

Minimal depth variable selection method differs from the traditional method of variable selection in RSF, which has been based on variable importance measures (Ishwaran et al., 2011). Minimal depth assesses the predictive ability of a variable by its depth relative to the root node of a tree. To determine the minimal depth, first define the maximal subtree for a variable vas the largest subtree whose root node is split using $v$ (the 
first-order maximal subtree). The shortest distance from the root of the tree to the root of the closest maximal subtree of $v$ is the minimal depth of $v$. The minimal depth can be considered as the minimal number of nodes crossed from the root node, until a split involving $v$ is encountered. A smaller value corresponds to a more predictive variable.

In high-dimensional settings, the distribution of the minimal depth can be used as a means to screen unimportant variables. The mean of the minimal depth distribution represents a threshold value for selecting variables, such that those variables with forest averaged minimal depth exceeding the mean minimal depth threshold are classified as noisy and removed from the final model.

The concept of maximal subtrees can be extended to second-order subtrees, to provide a powerful tool for identifying relationships between variables. A second-order maximal $(w, v)$-subtree is a maximal $w$-subtree within a maximal $v$-subtree for a variable $v$. In this way, we can quantify the association between two variables. Two-way interactions can be estimated by using forest averaged second-order maximal subtrees.

\subsection{Breast Cancer Data}

The genetic data usedin this thesis come from 441 specimens collected between 1989 and 1998 fromCaucasian women selected from the Hormone Receptor Laboratory (HRL) Biorepository and Tumor Marker Database (TMD)(Kidd et al., 2010). Human tissue specimens were collected from 235 node-negative and 206 node-positive patients.

The HRL Biorepository and the TMD contain de-identified specimens of breast carcinoma with associated tumor clinical outcome with up to 15 years of follow-up. Clinico-pathological data, such as tumor-based properties (e.g., pathology, grade, stage, 
size, tumor maker status), patient-related characteristics (e.g., age, race, menopausal status, family history, nodal status), and clinical follow-up (e.g., treatment regimen, DFS, OS) was available. Biochemical data, such as estrogen/progesterone receptor, epidermal growth factor receptor, and human epidermal growth factor receptor status were obtained from TMD.

DNA was isolated from this tissue using the AllPrep DNA/RNA/Protein Mini Kit (Qiagen, Valencia, CA) or QIAamp DNA Mini Kit (Qiagen). Thirteen angiogenesisrelated SNPs with minor allele frequency $>0.05$ were selected for our genetic analysis. In Table 1, we list the gene names, RefSNP (rs) numbers, and nucleotide change information for these thirteen SNPs.

TABLE 1.Selected SNPs in the angiogenesis pathway and their nucleotide change

\begin{tabular}{|c|c|c|}
\hline Gene & rs Number & Nucleotide change \\
\hline IL10_1082 & rs 1800896 & $\mathrm{G}>\mathrm{A}$ \\
\hline IL10_819 & rs1800871 & $\mathrm{C}>\mathrm{T}$ \\
\hline IL10_592 & rs 1800872 & $\mathrm{C}>\mathrm{A}$ \\
\hline IL-10R_109 & rs9610 & $\mathrm{G}>\mathrm{A}$ \\
\hline TGFb1_896 & rs1982073 & $\mathrm{T}>\mathrm{C}$ \\
\hline TGFbR1_195 & rs868 & $\mathrm{A}>\mathrm{G}$ \\
\hline VEGF_2578 & rs699947 & $\mathrm{C}>\mathrm{A}$ \\
\hline VEGF_1154 & rs 1570360 & $\mathrm{~A}>\mathrm{G}$ \\
\hline VEGF_634 & Rs2010963 & $\mathrm{G}>\mathrm{C}$ \\
\hline VEGFR_889 & rs2305948 & $\mathrm{G}>\mathrm{A}$ \\
\hline VEGFR_1416 & rs1870377 & $\mathrm{T}>\mathrm{A}$ \\
\hline VEGFR.IVS25_92 & rs1531289 & $\mathrm{G}>\mathrm{A}$ \\
\hline VEGFR.IVS6_54 & rs7692791 & $\mathrm{A}>\mathrm{G}$ \\
\hline
\end{tabular}

\subsection{Missing values}

Our approach for treating missing data in Lasso, Elastic Net, and Survival MDR methods was to plug in the most frequent genotype for each SNP for the subjects with 
missing values. RSF uses an adaptive tree imputation algorithm to adaptively impute missing values as the tree is grown. Missing data are imputed by using a set of randomly drawn observations from the set of non-missing in-bag data within the current node (Ishwaran et al., 2008). 


\section{CHAPTER IV}

\section{RESULTS}

\subsection{Penalized Cox regression models}

In order to fit penalized Cox regression models, we treat the 13 SNPs as categorical factors. For each of the 13 SNPs there are three different genotypes each with associated indicator variables, resulting in a total of 39 potential main effect terms. We describe $k$-way $(k=1,2,3)$ interaction models that capture the relationship between $k$ genes and the survival outcome. One-way interaction models include only main effect terms, while two-way interaction models include both main effects and all two-way interaction terms, and three-way interaction models include all main effect, two-way interaction, and three-way interaction terms. These full models are used as starting points for fitting the penalized regression models, which will eliminate some proportion of the terms depending on the magnitude of the shrinkage coefficient(s).

\subsection{1 $\mathrm{L}_{1}$-penalized Cox regression}

Table 2 summarizes the optimal tuning parameter $\lambda_{1}$ and corresponding crossvalidated log likelihood value based on the Lasso method, for the main effect, two-way, 
and three-way interaction models. As these results show, for each model there's an optimal tuning parameter $\lambda_{1}$ and a cross-validated log likelihood value.

TABLE 2.Optimal tuning parameter for Lasso method

\begin{tabular}{ccc}
\hline & $\lambda_{1}$ & $C V_{\mathrm{LL}}$ \\
\hline Main effect & 14.741 & -813.657 \\
Two-way & 10.802 & -812.737 \\
Three-way & 10.510 & -812.471 \\
\hline
\end{tabular}

$\mathrm{CV}_{\mathrm{LL}}$, cross-validated log likelihood

In Table 3, we summarize the selected one-, two-, and three-way models based on the Lasso method by listing the corresponding coefficients. To determine the most important SNPs for each model, we rank the coefficients by their absolute magnitude, with the largest magnitude coefficients ranked the highest. For the main effect model, no SNPs were included in the model. An interaction between IL10R_109_G/A and TGFBR1_195_A/A is selected as the most important coefficient from the two-way interaction model, and an interaction among VEGF_2578C/C, VEGFR_889G/G and VEGFR_1416T/T is the strongest coefficient from the three-way interaction model.

TABLE 3.Selected models based on Lasso method

\begin{tabular}{cccc}
\hline SNP & MainEffect & Two-way & Three-way \\
\hline VEGF_2578C/C & -- & 0.026 & -- \\
IL10R_109G/A:TGFBR1_195A/A & -- & -0.235 & -0.247 \\
VEGF_2578C/C:VEGFR_1416T/T & -- & 0.048 & -- \\
VEGFR.IVS25_92G/G:VEGFR.IVS6_54A/G & -- & -0.140 & -0.149 \\
IL10R_109G/G:VEGF_1154G/G:VEGFR_1416T/T & -- & -- & 0.024 \\
VEGF_2578C/C:VEGFR_889G/G:VEGFR_1416T/T & -- & 0.026 & -- \\
\hline Results are presented as coefficients. & & &
\end{tabular}


In Figure 3, we plot the survival curves for the high-risk versus low-risk genotypes based on the Lasso two-way and three-way models. For this method, we divide genotypes into high or low risk groups based on linear predictors. For each linear predictor, if its value exceeds the median of all linear predictors, then it is considered high-risk. Otherwise, it is considered low-risk.

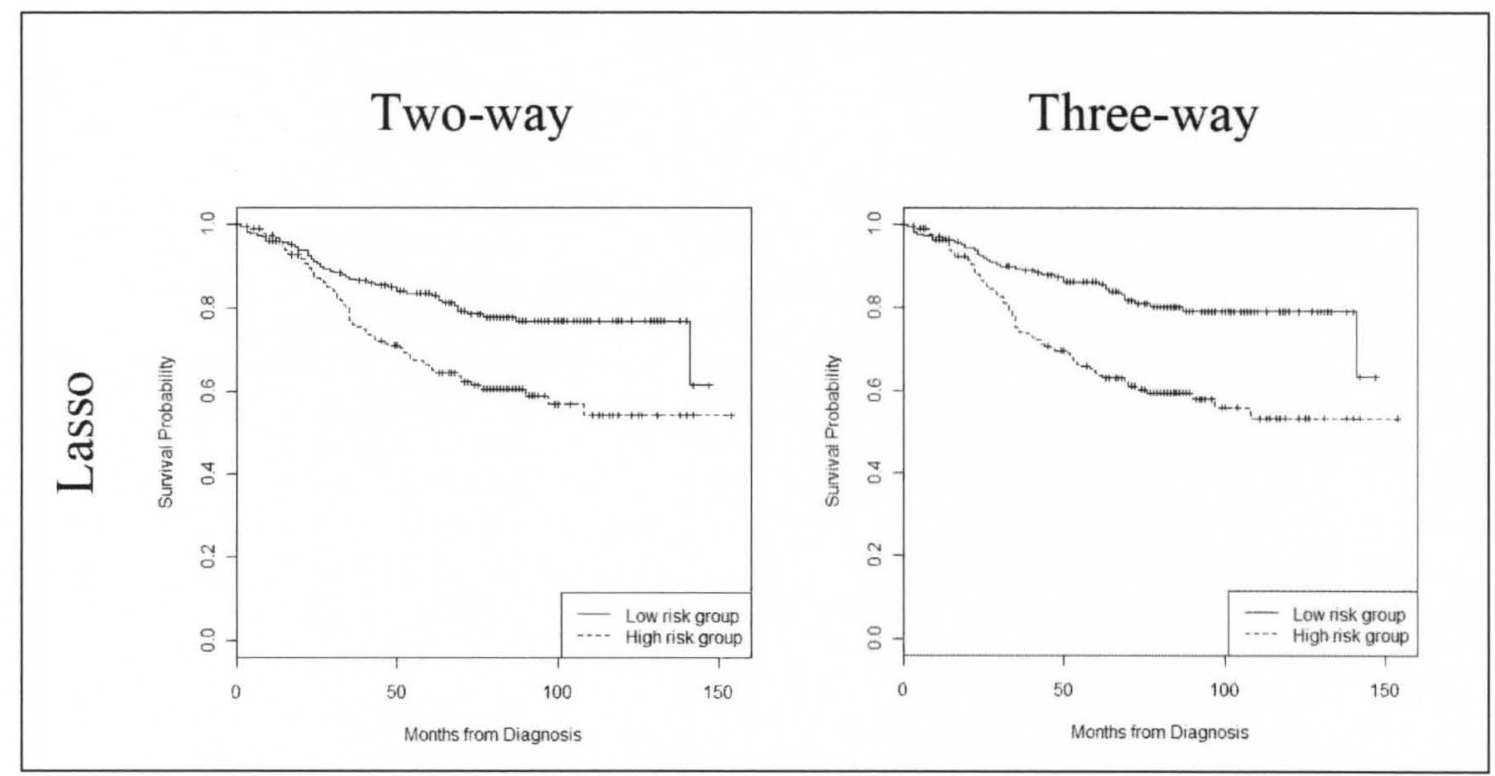

Fig.3 Kaplan-Meier survival curve for high and low risk groups based on Lasso two-way and three-way models

\subsubsection{Elastic Net}

Table 4 summarizes the optimal tuning parameters $\lambda_{1}$ and $\lambda_{2}$, and the corresponding cross-validated log likelihood values based on the Elastic Net method for the main effect, two-way, and three-way interaction models.

In Table 5, we summarize the top one, two, and three-way models identified by the Elastic Net. VEGF_2578_C/C is selected as the most important for main effects. A 
combination of IL10R_109_G/A and TGFBR1_195_A/A is selected as the most important two-way model. This combination is also selected as the most important threeway model.

TABLE 4.Optimal tuning parameter for Elastic Net method

\begin{tabular}{cccc}
\hline & $\lambda_{1}$ & $\lambda_{2}$ & $\mathrm{CV}_{\mathrm{LL}}$ \\
\hline Main effect & 8.031 & 0.01 & -813.413 \\
Two-way & 11.068 & 0.0001 & -813.461 \\
Three-way & 13.531 & 0.0001 & -813.561 \\
\hline
\end{tabular}

$\mathrm{CV}_{\mathrm{LL}}$, cross-validated log likelihood

TABLE 5.Selected models based on Elastic Net method

\begin{tabular}{cccc}
\hline SNP & MainEffect & Two-way & Three-way \\
\hline IL10R_109G/A & & & \\
IL10R_109G/G & -0.063 & -- & -- \\
VEGF_2578C/C & 0.027 & -- & -- \\
VEGFR.IVS25_92G/G & 0.167 & 0.023 & -- \\
VEGFR.IVS6_54A/G & -0.047 & -- & -- \\
IL10R_109G/A:TGFBR1_195A/A & -0.047 & -- & -- \\
VEGF_2578C/C:VEGFR_1416T/T & -- & -0.224 & -0.132 \\
VEGFR.IVS25_92G/G:VEGFR.IVS6_54A/G & -- & 0.035 & -- \\
\hline
\end{tabular}

Results are presented as coefficients.

In Figure 4, we plot the survival curves for the high-risk versus low-risk genotypes defined by Elastic Net method. We divide genotypes into high or low risk groups based on linear predictors. 


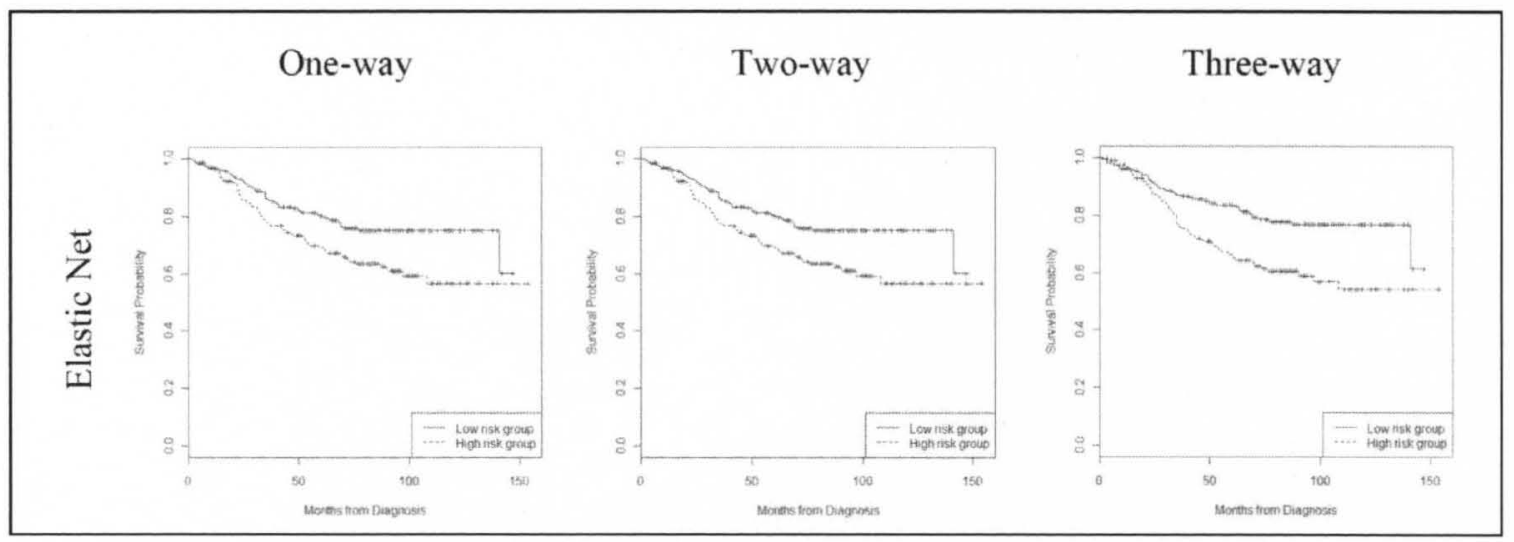

Fig.4 Kaplan-Meier survival curves for high and low risk groups based on Elastic Net one-, two-, and three-way models.

\subsection{Survival MDR}

For the main effects, two-way and three-way interaction combinations, the best models are shown in Table 6. The VEGF_2578 has the highest cross-validation consistencies $(60 \%)$ of the one factor model, and is therefore selected as the final best models. A combination between IL10R_109 and TGFBR1_195 serves as the best two factor predictors, with a cross validation consistency of $50 \%$. Lastly, a combination among IL10R_109, TGFBR1_195 and VEGF_2578 serve as the best three factor model with a cross validation consistency of $40 \%$. One curious result is that a main effect model (VEGFR.IVS25_92) appears amongst the list of models for both the two-way and threeway interaction evaluations, with a CVC of $10 \%$ in each case. We are unable to determine why this single factor model appeared in each case.

TABLE 6.Selected models based on Survival MDR method 


\begin{tabular}{cccc}
\hline SNP & MainEffect & Two-way & Three-way \\
\hline VEGF_2578 & $60 \%$ & -- & -- \\
VEGFR.IVS25_92 & $20 \%$ & $10 \%$ & $10 \%$ \\
VEGFR.IVS6_54 & $20 \%$ & - & -- \\
IL10R_109:TGFBR1_195 & -- & $50 \%$ & -- \\
IL10R_109:VEGF_1154 & -- & $40 \%$ & -- \\
IL10R_109:TGFBR1_195:VEGF_2578 & -- & -- & $40 \%$ \\
IL10R_109:TGFBR1_195:VEGF_1154 & -- & -- & $20 \%$ \\
IL10R_109:VEGF_1154:VEGFR_1416 & -- & -- & $10 \%$ \\
VEGF_2578:VEGFR.IVS25_92:VEGFR.IVS6_54 & -- & -- & $10 \%$ \\
VEGF_1154:VEGFR.IVS25_92:VEGFR.IVS6_54 & -- & -- & $10 \%$ \\
\hline
\end{tabular}

Results are presented as cross-validated consistency (cvc).

Every genotype is assigned into an appropriate group (high / low risk) by Survival MDR's constructive induction algorithm. Based on this, we constructed Kaplan-Meier plots of the high and low risk groups determined by the best one-, two-, and three-way models (Figure 5).

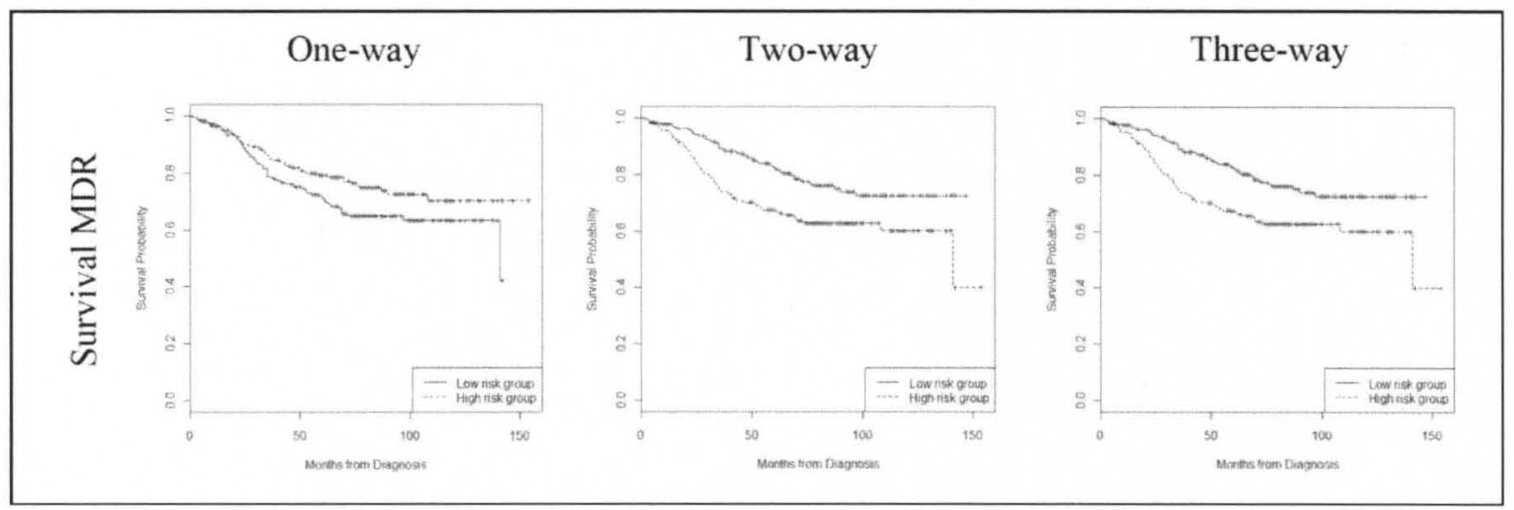

Fig.5 Kaplan-Meier survival curve for high and low risk groups based on Survival MDR one-, two-, and three-way models.

\subsection{Random Survival Forest}


Table 7 gives the average VIMP scores and minimal depth thresholds for each SNP based on 50 independent runs of RSF with mtry equal to 1000 . Both the VIMP and minimal depth selection methods select IL10R_109 as the most important main effect. The second order minimal depth values for each SNP relative to the other SNPs were calculated, and these values were used to define a distance matrix between the SNPs. Figure 6 plots the resulting dendrogram based on hierarchical clustering of the data. SNPs IL10_1082 and IL10R_109 are joined together first based on the hierarchical clustering, and indicated a possible strong interaction between the SNP pair.

TABLE 7.Selected models based on Random Survival Forest method

\begin{tabular}{cccccc}
\hline & VIMP & \multicolumn{3}{c}{ Minimal Depth } \\
Variable & Average & Rank & Variable & Average & Rank \\
\hline IL10R_109 & 0.035 & 1.00 & IL10R_109 & 0.043 & 1.00 \\
VEGF_2578 & 0.014 & 2.68 & VEGF_2578 & 0.015 & 3.07 \\
VEGF_634 & 0.011 & 3.54 & VEGF_634 & 0.014 & 3.23 \\
VEGF_1154 & 0.009 & 4.42 & TGFBR1_195 & 0.013 & 3.44 \\
TGFBR1_195 & 0.009 & 4.84 & TGFB1_896 & 0.009 & 4.83 \\
TGFB1_896 & 0.006 & 5.96 & VEGF_1154 & 0.005 & 6.45 \\
IL10_592 & 0.003 & 7.46 & IL10_592 & 0.002 & 7.32 \\
VEGFR.IVS6_54 & 0.002 & 7.54 & VEGFR_1416 & 0.002 & 7.56 \\
VEGFR.IVS25_92 & 0.000 & 8.94 & VEGFR.IVS6_54 & -0.002 & 9.52 \\
VEGFR_1416 & -0.002 & 9.92 & IL10_1082 & -0.004 & 10.69 \\
IL10_1082 & -0.004 & 10.92 & IL10_819 & -0.005 & 11.13 \\
VEGFR_889 & -0.005 & 11.46 & VEGFR_889 & -0.005 & 11.25 \\
IL10_819 & -0.007 & 12.32 & VEGFR.IVS25_92 & -0.005 & 11.51 \\
\hline
\end{tabular}




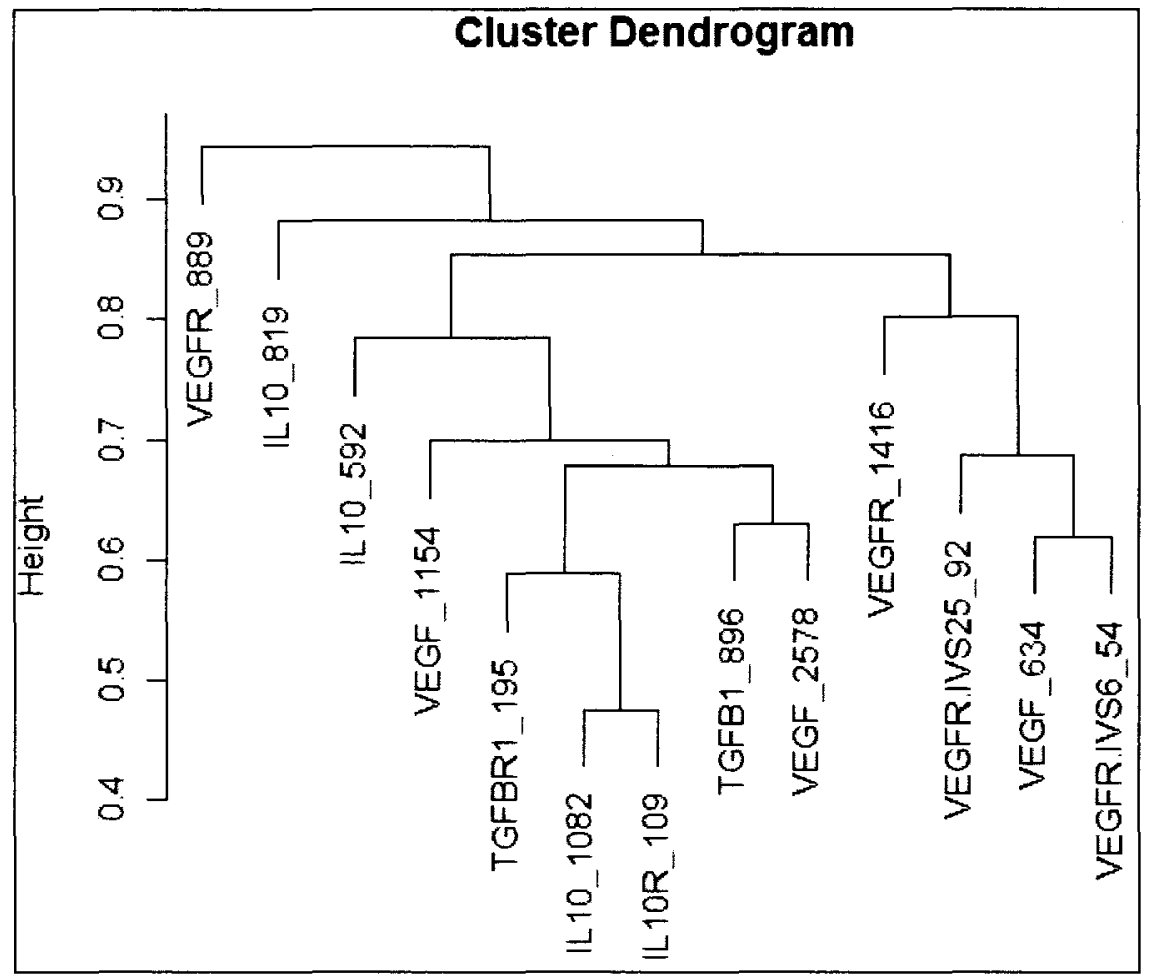

Fig.6 Variables clustered by second-order minimal depth

\subsection{Comparison of Results}

As shown in Tables 8,9 , and 10, we list the two most important SNPs from the one, two, and three-way models identified by Lasso, Elastic Net, and Survival MDR. Also given are the most important SNPs based on the VIMP and minimal depth from RSF, and the most significant two-way interaction identified by minimal depth using second-order maximal subtrees. In Figure 8, we put the Kaplan-Meier survival curves based on the Lasso, Elastic Net and Survival MDR methods together. 
Table 8. Comparison table of significant variables selected by different methods based on main effect models.

\begin{tabular}{cc}
\hline Method & Main Effect \\
\hline Lasso & -- \\
& -- \\
Elastic Net & VEGF_2578C/C \\
& IL10R_109G/G \\
SurvivalMDR & VEGF_2578/VEGFR.IVS25_92 \\
& VEGFR.IVS6_54 \\
VIMP & IL10R_109 \\
& VEGF_2578 \\
MinimalDepth & IL10R_109 \\
& VEGF_2578 \\
\hline
\end{tabular}

Table 9. Comparison table of significant variables selected by different methods based on two-way models.

\begin{tabular}{cc}
\hline Method & Two-way \\
\hline Lasso & IL10R_109G/A:TGFBR1_195A/A \\
& VEGFR.IVS25_92G/G:VEGFR.IVS6_54A/G \\
Elastic Net & IL10R_109G/A:TGFBR1_195A/A \\
& VEGFR.IVS25_92G/G:VEGFR.IVS6_54A/G \\
SurvivalMDR & IL10R_109: TGFBR1_195 \\
& IL10R_109: VEGF_1154 \\
VIMP & -- \\
& -- \\
MinimalDepth & IL10_1082:IL10R_109 \\
& VEGF_634:VEGFR.IVS6_54 \\
\hline
\end{tabular}


Table 10. Comparison table of significant variables selected by different methods based on three-way models.

\begin{tabular}{cc}
\hline Method & Three-way \\
Lasso & IL10R_109G/A:TGFBR1_195A/A \\
& VEGF_2578C/C:VEGFR_889G/G:VEGFR_1416T/T \\
Elastic Net & IL10R_109G/A:TGFBR1_195A/A \\
& VEGFR.IVS25_92G/G:VEGFR.IVS6_54A/G \\
SurvivalMDR & IL10R_109: TGFBR1_195: VEGF_2578 \\
& IL10R_109:TGFBR1_195:VEGF_1154 \\
VIMP & -- \\
& -- \\
MinimalDepth & -- \\
& -- \\
\hline
\end{tabular}

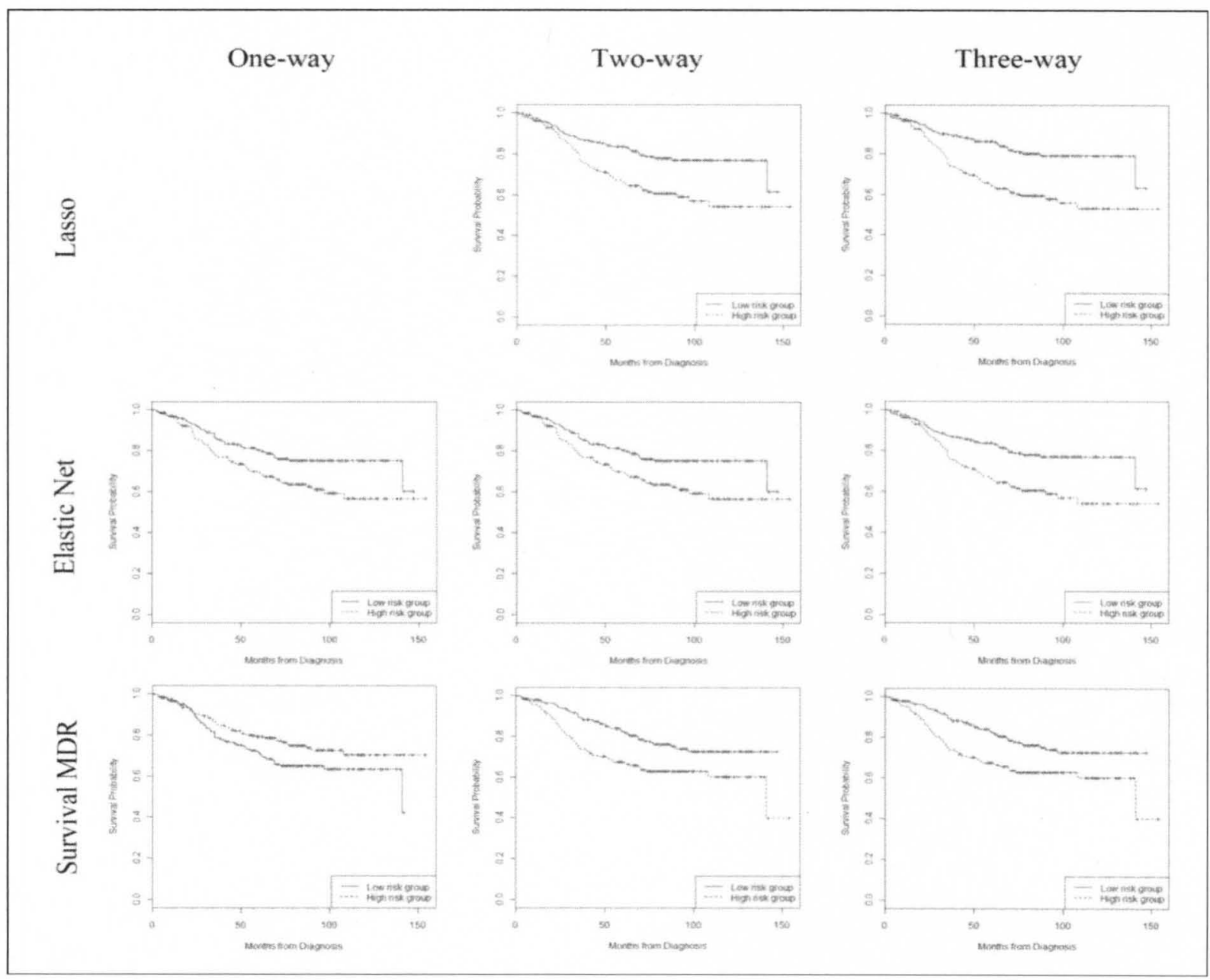


Fig. 8 Kaplan-Meier survival curve for high and low risk groups based on Lasso, Elastic Net, and Survival MDR methods.

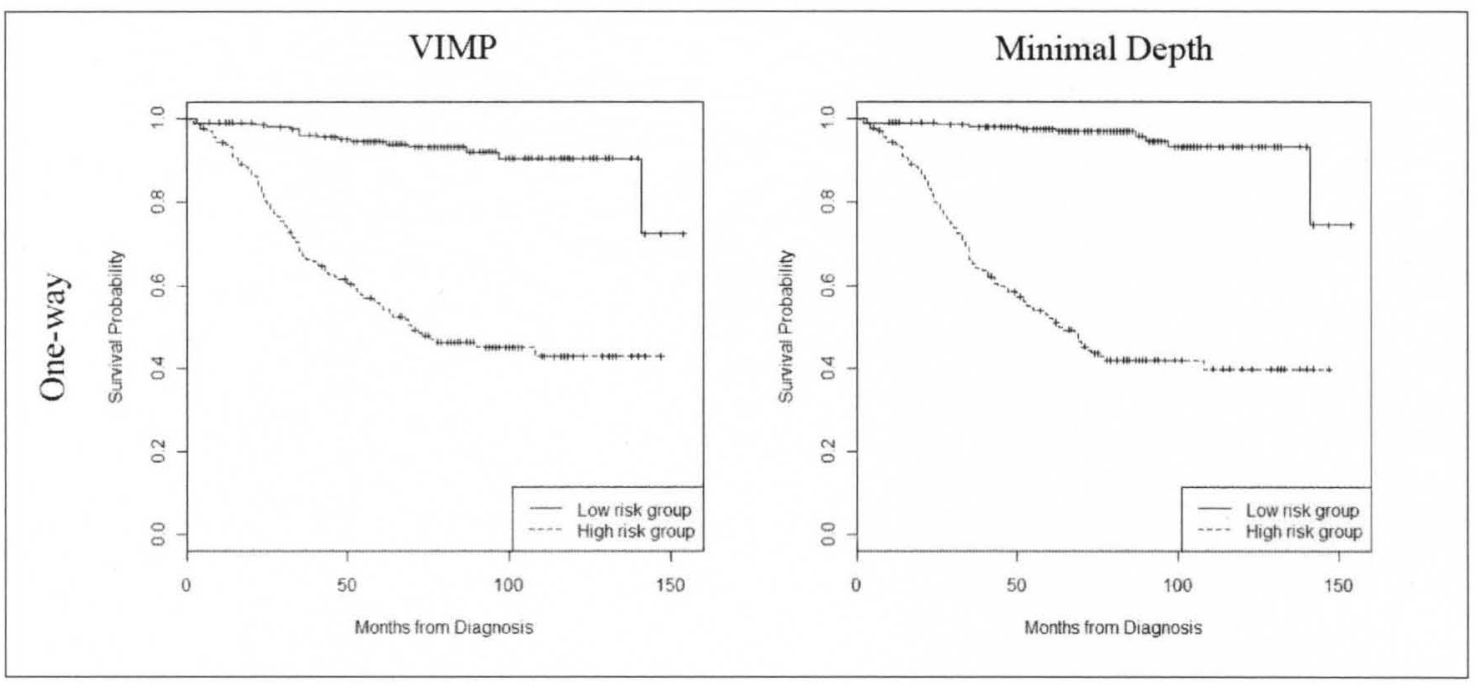

Fig. 9 Kaplan-Meier survival curve for high and low risk groups based on VIMP and minimal depth one-way models

In Figure 9, we plot the Kaplan-Meier survival curves for high and low risk groups based on the VIMP and minimal depth models. In this situation, we separate high or low risk groups according to VIMP score and mean minimal depth threshold. We use median as the threshold. Note that, as opposed to the other methods, there are no additional two- and three-way interaction models for the RSF method. This is because RSF already implicitly includes interactions, in the sense that second-order maximal $(w, v)$-subtrees identify interactions between two variables $w$ and $v$. 


\section{CHAPTER V}

\section{DISCUSSION}

In the current study, we directly compared the performance of four variable selection techniques for detecting SNP-SNP interactions in survival genetic association studies: the Lasso and Elastic Net penalized Cox regression models, Survival Multifactor Dimensionality Reduction, and Random Survival Forest. As shown in the previous section, we demonstrate that all these methods can detect the presence of multiplicative interaction models even when the main effects are not statistically significant. All of the plots shown in the previous section indicate the significant SNPs chosen by these four methods separate the two survival curves, although the statistical significant of this separation was not evaluated.

Among the four methods, the Lasso and Elastic Net are semi-parametric methods. The Elastic Net performed very similarly to the Lasso in our study, particularly for the two-, and three-way interaction models. Both methods select the same top two most important terms in each case. But the additional $\mathrm{L}_{2}$-penalty for the Elastic Net distributes the weight to more variables, so that the Elastic Net selected more variables overall compared to the Lasso. This is especially useful in the situation of high correlation. When high correlation exists, the Lasso will only pick one of the correlated variables, whereas the Elastic Net will select the entire set of correlated variables. Thus, it is not surprising 
to see that the Lasso selected no main effect terms, whereas five SNPs were selected using the Elastic Net. A limitation of these two methods is that they constructed and selected variables corresponding to individual genotypes, rather than selecting the set of genotypes corresponding to a particular SNP. This shortcoming can be overcome by extending the group Lasso to survival time data (Meier et al., 2008; Winham et al., 2011). In contrast, Survival MDR and Random Survival Forest are non-parametric methods. The potential advantage of Survival MDR over parametric methods lies in determining high and low risk groups for genotype combinations regardless of whether linear relationships exist between survival time and SNP effects. A limitation of Survival MDR is that it does not have a way to adjust for covariate effects such as gender, family history, and smoking status. In terms of Random Survival Forest, it is highly data adaptive and its computation is fast even for very large data sets. It can adaptively impute missing data as a tree is grown using its own missing data algorithm. This might cause difference between the result of RSF and other methods since they are using different ways in imputing missing values. As shown in the previous section, both the Elastic Net and Survival MDR select VEGF_2578 $\mathrm{C} / \mathrm{C}$ as the most important main effect term. However, both the VIMP and minimal depth choose IL10R_109 as the most important main effect. Different results also show up in the situation of the most important two-way interactions.

In recent years, some researchers have attempted to evaluate the performance of different approaches to analyze high-dimensional data with respect to a survival endpoint. Benner and Zucknick (Benner et al., 2010) implemented the Lasso, Elastic Net, adaptive Lasso, and smoothly clipped absolute deviation (SCAD) methods into the model building 
process when analyzing high-dimensional data with the Cox proportional hazards model. They demonstrated that the Lasso and also Elastic Net performed the best in terms of prediction accuracy, and thus recommended to use those approaches in actual data application. Winham (Winham et al., 2011) compared the performance of MDR, the traditional Lasso with $\mathrm{L}_{1}$ penalty, and the group Lasso for categorical data with groupwise $L_{1}$ penalty to detect gene-gene interactions. Unlike Benner, they evaluated the predictive performance using a broad range of simulations. They found that the performance of each method is context dependent. Thus, they concluded that each approach might be best suited for detecting and characterizing interactions with different mechanisms. Similarly to Winham, we found differences between the methods when identifying the most prognostic relevant covariates.

While this study is useful for informing researchers about different analysis tools for identifying SNP-SNP interactions, it is not a fully comprehensive comparison. In order to definitely guide researchers in how to choose an analysis method for real data application, prediction error for each method on independent test data should be provided. In addition, various difficulties in real data such as missing values and genetic heterogeneity should be incorporated into the comparison. Additional methods not investigated here may prove even more fruitful for identifying SNP-SNP interactions. For example, the adaptive Lasso has proved a promising technique with attractive theoretical properties. We anticipate that these methods will be used to find interactions among covariate effects, in addition to genotypes. We also anticipate comparing the performance of these methods to detect gene-gene interactions through simulations. 
In summary, we compared and contrasted the performance of four variable selection approaches to identify epistatic interactions between SNPs using survival data for breast cancer patients. While we have not conducted a comprehensive comparison in order to determine which method(s) performed the best for indentifying interactions, each method has potential for application to high-dimensional genetic interaction data with survival outcomes.. 


\section{REFERENCES}

Benner, A., Zucknick, M., Hielscher, T., Ittrich, C., and Mansmann, U. (2010). High-dimensional Cox models: the choice of penalty as part of the model building process. Biom J 52, 50-69.

Breiman, L. (2001). Random forests. Mach Learn 45, 5-32.

Breslow, N. E. Analysis of Survival Data under the Proportional Hazards Model. International Statistical Review 43 (1975): 45-58.

Cox, D. R. Regression Model and Life Tables (with Discussion). Journal of the Royal Statistical Society B 34 (1972) : 187-220.

Datta, S., Le-Rademacher, J., and Datta, S. (2007). Predicting patient survival from microarray data by accelerated failure time modeling using partial least squares and LASSO. Biometrics 63 , 259-271.

Gui, J., Moore, J.H., Kelsey, K.T., Marsit, C.J., Karagas, M.R., and Andrew, A.S. (2011). A novel survival multifactor dimensionality reduction method for detecting gene-gene interactions with application to bladder cancer prognosis. Hum Genet 129, 101-110.

Ishwaran, H., Kogalur, U.B., Blackstone, E.H., and Lauer, M.S. (2008). Random Survival Forests. Ann Appl Stat 2, 841-860.

Kaplan, E.L. and Meier, P. Nonparametric Estimation from Incomplete Observations. Journal of the American Statistical Association 53 (1958): 457-481.

Kidd, L.R., Brock, G.N., VanCleave, T.T., Benford, M.L., Lavender, N.A., Kruer, T.L., and Wittliff, J.L. (2010). Angiogenesis-associated sequence variants relative to breast cancer recurrence and survival. Cancer Causes Control 21, 1545-1557.

Lecessie, S., and Vanhouwelingen, J.C. (1992). Ridge Estimators in Logistic-Regression. Appl StatJ Roy St C 41, 191-201.

Meier, L., van de Geer, S.A., and Buhlmann, P. (2008). The group lasso for logistic regression. J Roy Stat Soc B 70, 53-71.

Ritchie, M.D., Hahn, L.W., Roodi, N., Bailey, L.R., Dupont, W.D., Parl, F.F., and Moore, J.H. (2001). Multifactor-dimensionality reduction reveals high-order interactions among estrogenmetabolism genes in sporadic breast cancer. Am J Hum Genet 69, 138-147. 
Tibshirani, R. (1997). The lasso method for variable selection in the Cox model. Stat Med 16, 385-395.

Verweij, P.J., and Van Houwelingen, H.C. (1994). Penalized likelihood in Cox regression. Stat Med $13,2427-2436$.

Winham, S., Wang, C., and Motsinger-Reif, A.A. (2011). A comparison of multifactor dimensionality reduction and L1-penalized regression to identify gene-gene interactions in genetic association studies. Stat Appl Genet Mol Biol 10, Article 4.

Witten, D.M., and Tibshirani, R. (2010). Survival analysis with high-dimensional covariates. Stat Methods Med Res 19, 29-51.

Zou, H., and Hastie, T. (2005). Regularization and variable selection via the elastic net. J Roy Stat Soc B 67, 301-320. 


\section{APPENDIX}

\section{$R$ code for data management}

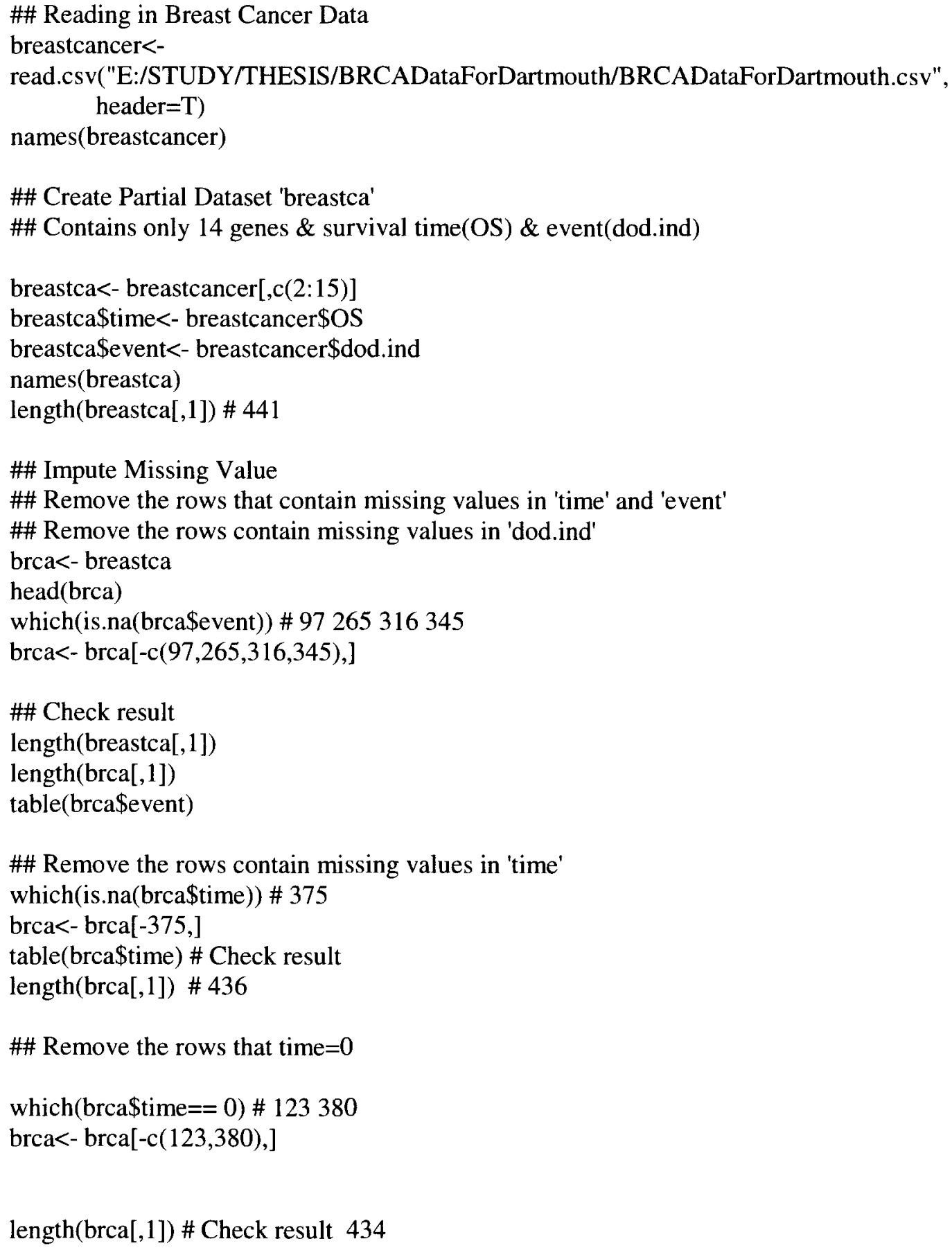


\#\# Assign all missing samples using the most common genotype

brca.mean[,1][which(is.na(brca.mean[,1]))]<-geno

table(brca.mean[,1])

\#\# Try to use a LOOP through all the genes (1-14)

for(i in 1:14) 1

tab $<-$ table(brca.mean[,i])

geno<- names(tab) [which.max (table(brca.mean[,i]))]

\}

brca.mean[,i][which(is.na(brca.mean[,i]))]<-geno

\#\#brca.mean \# Check the result

length(brca.mean[,1]) \#434

\#\# Convert 1st Dataset Factors to Numbers

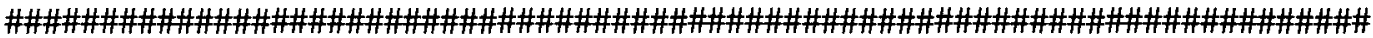

\#\# Create separate data.frame called 'numeric brca'

\#\# Levels should be $0,1,2$ (1= heterozygote, $0=$ most common homozygous)

numeric.brca.mean<- brca.mean

names(numeric.brca.mean)

\#\# "IL10_1082"

length(which(is.na(numeric.brca.mean[,1]))) \# Check again

table(numeric.brca.mean[,1])

\#\# A/A A/G G/G

\#\# 116201117

numeric.brca.mean[,1]<-

ifelse(numeric.brca.mean[,1]\%in\%" $A / G ", 1$,ifelse(numeric.brca.mean[,1]\%in\%"G/G",0,2)) table(numeric.brca.mean[,1]) \# check result

\#\# "IL10_819"

table(numeric.brca.mean[,2])

\#\# $\mathrm{C} / \mathrm{C} \mathrm{C} / \mathrm{T} \mathrm{T} / \mathrm{T}$

\#\# 30410921

numeric.brca.mean[,2]<-

ifelse(numeric.brca.mean[,2]\%in\%"C/T",1,ifelse(numeric.brca.mean[,2]\%in\%"C/C",0,2)) table(numeric.brca.mean[,2]) \# check result

\#\# "IL10_592"

table(numeric.brca.mean[,3])

\#\# A/A A/C C/C

\#\# 13117304

numeric.brca.mean[,3]<-

ifelse(numeric.brca.mean[,3]\% in\%"A/C", 1, ifelse(numeric.brca.mean[,3]\%in\%"C/C",0,2)) table(numeric.brca.mean[,3]) \# check result 
\#\# "IL10R_241"

table(numeric.brca.mean[,4])

\#\# A/A A/G G/G

\#\# 58148228

numeric.brca.mean[,4]<-

ifelse(numeric.brca.mean[,4]\% in\%"A/G",1,ifelse(numeric.brca.mean[,4]\%in\%"G/G",0,2)) table(numeric.brca.mean[,4]) \# check result

\#\# "IL10R_109"

table(numeric.brca.mean[,5])

\#\# A/A G/A G/G

\#\# 74216144

numeric.brca.mean[,5]<-

ifelse(numeric.brca.mean[,5]\% in\%"G/A",1, ifelse(numeric.brca.mean[,5]\%in\%"G/G",0,2)) table(numeric.brca.mean[,5]) \# check result

\#\# "TGFB1_896"

table(numeric.brca.mean[,6])

\#\# C/C C/T T/T

\#\#35236163

numeric.brca.mean[,6]<-

ifelse(numeric.brca.mean[,6]\%in\%"C/T",1, ifelse(numeric.brca.mean[,6]\%in\%"T/T",0,2))

table(numeric.brca.mean[,6]) \# check result

\#\# "TGFBR1_195"

table(numeric.brca.mean[,7])

\#\# A/A A/G G/G

\#\# 28613216

numeric.brca.mean[,7]<-

ifelse(numeric.brca.mean[,7]\%in\%"A/G",1,ifelse(numeric.brca.mean[,7]\%in\%"A/A",0,2)) table(numeric.brca.mean[,7]) \# check result

\#\# "VEGF_2578"

table(numeric.brca.mean[,8])

\#\# A/A C/A C/C

\#\# 85216133

numeric.brca.mean[,8]<-

ifelse(numeric.brca.mean[,8]\% in\%"C/A", 1, ifelse(numeric.brca.mean[,8]\%in\%"C/C",0,2)) table(numeric.brca.mean[,8]) \# check result

\#\# "VEGF_1154"

table(numeric.brca.mean[,9])

\#\# A/A A/G G/G

\#\# 24152258

numeric.brca.mean[,9]<-

ifelse(numeric.brca.mean[,9]\% in\%"A/G",1,ifelse(numeric.brca.mean[,9]\%in\%"G/G",0,2)) table(numeric.brca.mean[,9]) \# check result 
\#\# "VEGF_634"

table(numeric.brca.mean[,10])

\#\# C/C G/C G/G

\#\# 53217164

numeric.brca.mean[,10]<-

ifelse(numeric.brca.mean[,10]\%in\%"G/C",1,ifelse(numeric.brca.mean[,10]\%in\%"G/G",0,2)) table(numeric.brca.mean[,10]) \# check result

\#\# "VEGFR_889"

table(numeric.brca.mean[,11])

\#\# A/A A/G G/G

\#\# 581348

numeric.brca.mean[,11]<-

ifelse(numeric.brca.mean[,11]\%in\%"A/G",1, ifelse(numeric.brca.mean[,11]\%in\%"G/G",0,2)) table(numeric.brca.mean[,11]) \# check result

\#\# "VEGFR_1416"

table(numeric.brca.mean[,12])

\#\# A/A T/A T/T

\#\# 17120297

numeric.brca.mean[,12]<-

ifelse(numeric.brca.mean[,12]\%in\%"T/A",1, ifelse(numeric.brca.mean[,12]\%in\%"T/T",0,2)) table(numeric.brca.mean[,12]) \# check result

\#\# "VEGFR.IVS25_92"

table(numeric.brca.mean[,13])

\#\# A/A G/A G/G

\#\#31 127276

numeric.brca.mean[,13]<-

ifelse(numeric.brca.mean[,13]\% in\%"G/A",1,ifelse(numeric.brca.mean[,13]\%in\%"G/G",0,2)) table(numeric.brca.mean[,13]) \# check result

\#\# "VEGFR.IVS6_54"

table(numeric.brca.mean[,14])

\#\# A/A A/G G/G

\#\# 13321784

numeric.brca.mean[,14]<-

ifelse(numeric.brca.mean[,14]\% in\%"A/G",1, ifelse(numeric.brca.mean[,14]\%in\%"AA",0,2)) table(numeric.brca.mean[,14]) \# check result head(numeric.brca.mean) \# Check Convertion

\#\# remove snps that fail HWE "IL10R_241"

brca<- read.csv("brca.csv")

names(brca)

brca<- brca[,-c( 1,5$)]$ 
brca.mean<- read.csv("brca.mean.csv")

names(brca.mean)

brca.mean<- brca.mean[,-c $(1,5)]$

numeric.brca.mean<- read.csv("numeric.brca.mean.csv")

names(numeric.brca.mean)

numeric.brca.mean<- numeric.brca.mean[,-cc $(1,5)]$

\section{$R$ code for Lasso method}

library(penalized)

library(survival)

set.seed(101)

\#\# main effects

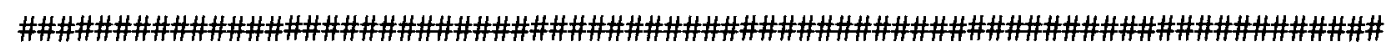

opt $1<-$ optL1 (Surv(brca.mean\$time, brca.mean\$event $) \sim$, data $=$ brca.mean[1:13], fold=10) opt 1 1 lambda \# 14.74060

opt 1 cvl \#cross-validation likelihood= -813.6569

\#\# Search for best one way model

fit $1<-$ penalized(Surv(brca.mean\$time, brca.mean\$event $) \sim$, data = brca.mean[1:13],

coefficients(fit1)

$$
\text { lambda } 1=14.74060, \text { lambda } 2=0 \text { ) }
$$

\#\# two way interactions

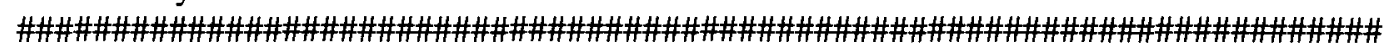

opt $2<-$ optL1(Surv(brca.mean\$time, brca.mean\$event $) \sim \wedge$, data $=$ brca.mean[1:13], fold=10) opt 2 \$ lambda \# 10.80183

opt $2 \$$ cvl \#cross-validation likelihood $=-812.7365$

\#\# Search for best two way model

fit $2<-$ penalized(Surv(brca.mean\$time, brca.mean\$event $) \sim$.^2, data = brca.mean[1:13],

coefficients(fit2)

$$
\text { lambda } 1=10.80183 \text {, lambda } 2=0 \text { ) }
$$

\#\# three way interactions

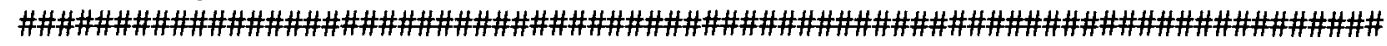

opt3 <- optL1(Surv(brca.mean\$time, brca.mean\$event $) \sim$.^3, data = brca.mean[1:13], fold=10) opt3\$ lambda \# 10.51043

opt3\$ cvl \#-812.4712

\#\# Search for best three way model

fit3 <- penalized(Surv(brca.mean $\$$ time, brca.mean\$event) $\sim$.^3, data = brca.mean[1:13], lambda $1=10.51043$, lambda $2=0$ ) 
coefficients(fit3)

\#\# Lasso KM curve based on three-way model

\#\# divide patients into low/high risk based on linear predictor

slotNames(fit3)

$\mathrm{lp}<$ - linear.predictors(fit3)

lp. $50<-$ median $(1 \mathrm{p})$

lp.low.high $<-$ ifelse $(l p<=1 p .50,0,1)$

fit.lp.2grps <- survfit(Surv(brca.mean\$time, brca.mean\$event) lp.low.high)

plot(fit.lp.2grps, main="Lasso KM curve based on three-way model", xlab = "Months from Diagnosis", ylab="Survival Probability", lty=1:2, cex=0.5)

legend("bottomright", c("Low risk group", "High risk group"), lty=1:2)

\section{$R$ code for Elastic Net method}

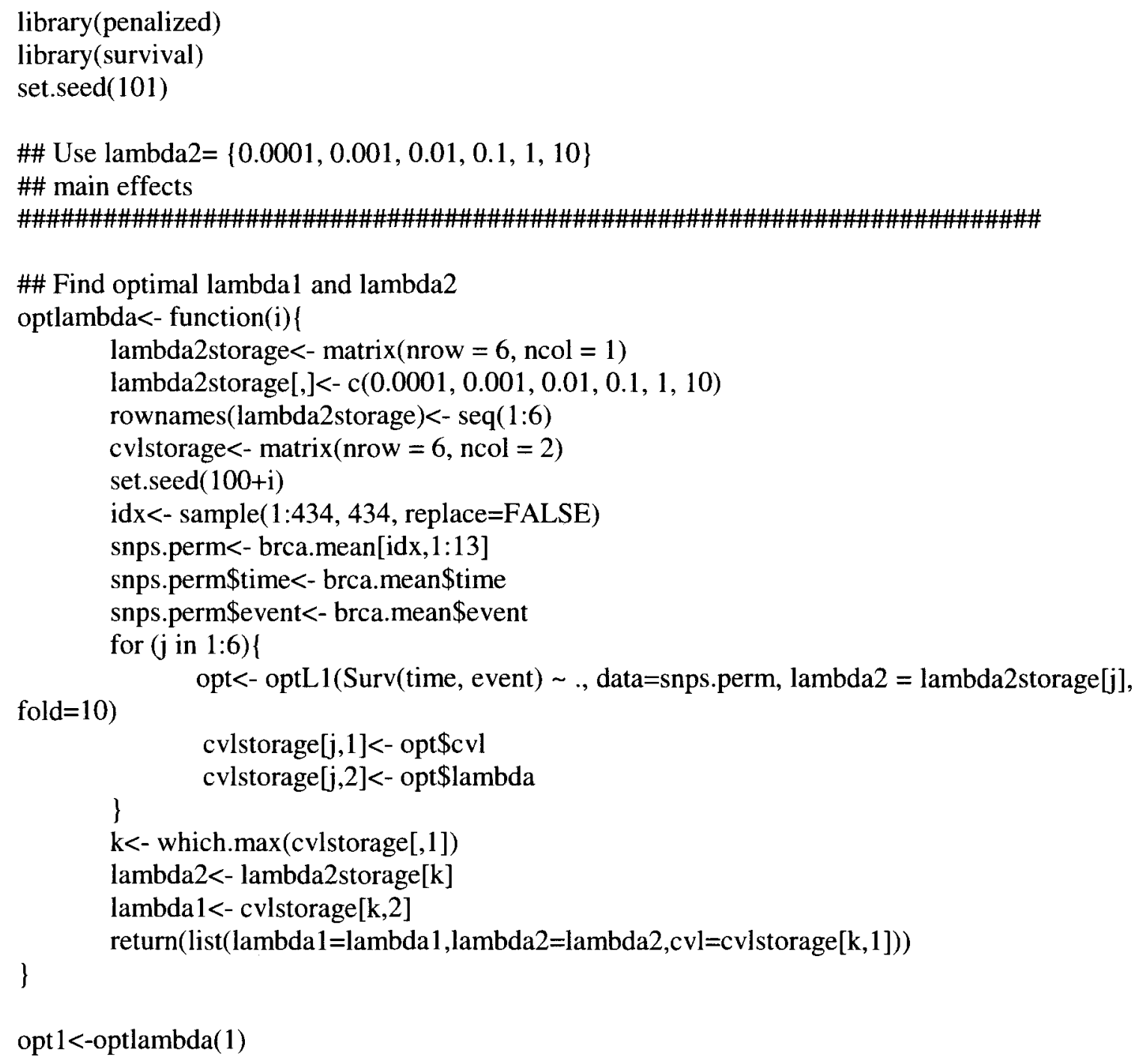


opt 1

fit $1<-$ penalized(Surv(brca.mean $\$$ time, brca.mean\$event $) \sim$., data $=$ brca.mean[1:13], lambda $1=8.0312$, lambda2 $=0.01$ )

coefficients(fit 1$)$

\#\# Interactions: $\sim .^{\wedge} 2$

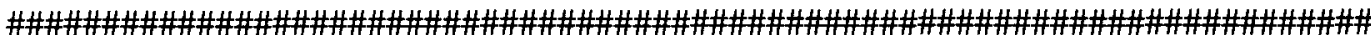

optlambda2<- function(i) \{

lambda2storage $<-$ matrix $($ nrow $=6, \mathrm{ncol}=1)$

lambda2storage[,]<- c $(0.0001,0.001,0.01,0.1,1,10)$

rownames(lambda2storage) $<-$ seq( $1: 6)$

cvlstorage $<$ - matrix $($ nrow $=6$, ncol $=2)$

set.seed $(100+i)$

idx $<-$ sample $(1: 434,434$, replace $=$ FALSE $)$

snps.perm<- brca.mean[idx,1:13]

snps.perm\$time<- brca.mean\$time

snps.perm\$event<- brca.mean\$event

for $(j$ in $1: 6)\{$

opt<- optL1(Surv(time, event) $\sim \wedge 2$, data=snps.perm, lambda $2=$

lambda2storage $[\mathrm{j}]$, fold $=10$ ) cvlstorage $[\mathrm{j}, 1]<-$ opt $\$ \mathrm{cvl}$

) cvlstorage $[\mathrm{j}, 2]<-$ opt $\$$ lambda

$\mathrm{k}<-$ which.max (cvlstorage[,1])

lambda2<- lambda2storage[k]

lambda $1<-$ cvlstorage $[\mathrm{k}, 2]$

return $($ list $($ lambda $1=$ lambda 1, lambda2=lambda2,cvl=cvlstorage $[k, 1]))$

\}

opt $2<-$ optlambda2(1)

opt2

\#\# optimal parameters: lambda1 $=9.053774$, lambda2 $=0.0001$

fit $2<-$ penalized(Surv(brca.mean $\$$ time, brca.mean\$event) $\sim .2$, data $=$ brca.mean[1:13], lambda $1=11.06831$, lambda2 $=0.0001$ )

summary(fit2)

coefficients(fit2)

\#\# Interactions: .^3

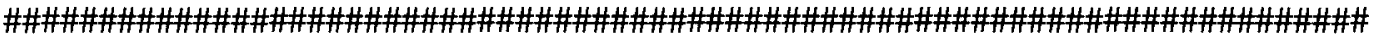

optlambda3<- function(i) \{

lambda2storage $<-$ matrix $($ nrow $=6$, ncol $=1)$

lambda2storage[, $]<-\mathrm{c}(0.0001,0.001,0.01,0.1,1,10)$

rownames(lambda2storage) $<-$ seq $(1: 6)$

cvlstorage $<$ - matrix $($ nrow $=6$, ncol $=2)$ 
set.seed(100+i)

idx $<-$ sample $(1: 434,434$, replace $=$ FALSE $)$

snps.perm<- brca.mean[idx, 1:13]

snps.perm\$time<- brca.mean\$time

snps.perm\$event $<-$ brca.mean\$event

for $(j$ in $1: 6)\{$

opt<- optL1(Surv(time, event) $\sim \wedge 3$, data=snps.perm, lambda $2=$ lambda2storage[j], fold $=10$ )

cvlstorage $[\mathrm{j}, 1]<-$ opt $\$ \mathrm{cvl}$

cvlstorage[j,2]<- opt\$lambda

\}

$\mathrm{k}<-$ which.max (cvlstorage[,1])

lambda2<- lambda2storage[k]

lambda $1<$ - cvlstorage[ $\mathrm{k}, 2]$

\}

return $($ list $($ lambda $1=$ lambda 1, lambda $2=$ lambda $2, \mathrm{cvl}=\mathrm{cvlstorage}[\mathrm{k}, 1]))$

\#\# lambda3<- optlambda3(1)

fit3 <- penalized(Surv(brca.mean\$time, brca.mean\$event) .^3, data = brca.mean[1:13],

coefficients(fit3) lambda $1=13.53075$, lambda $2=1 \mathrm{e}-04$ )

\#\# Elastic Net KM curve based on three-way model

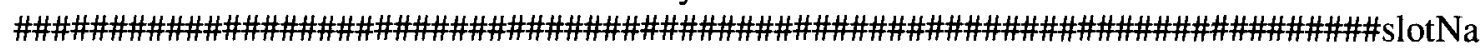

mes(fit)

$\mathrm{lp}<-$ linear.predictors(fit3)

lp. $50<-$ median $(l p)$

lp.low.high $<-$ ifelse $(l p<=1 p .50,0,1)$

fit.lp.2grps <- survfit(Surv(brca.mean\$time, brca.mean\$event) lp.low.high)

plot(fit.lp.2grps,main="Elastic Net KM curve based on three-way model", xlab = "Months from Diagnosis", ylab="Survival Probability", lty=1:2, cex=0.5)

legend("bottomright", c("Low risk group", "High risk group"), lty=1:2)

\section{$R$ code for Survival MDR method}

\#\# Survival MDR use data 'numeric.brca.mean'

source("Surv-MDR.R")

set.seed(101)

\#\# Search for the best one-way model

ffit $1<-$ ssimucross(numeric.brca.mean[1:13],numeric.brca.mean\$time, numeric.brca.mean\$event, 10,1) 
table(ffit 1 \$index)

\#\# 71213

\#\# 622

colnames(numeric.brca.mean)[c(7,12,13)] \# "VEGF_2578" "VEGFR.IVS25_92"

"VEGFR.IVS6_54"

\#\# Search for the best two-way model

ffit2 <- ssimucross(numeric.brca.mean[1:13],numeric.brca.mean\$time, numeric.brca.mean\$event, 10,2)

table(ffit 2 \$index)

\#\# 12406408

\#\# 155

colnames(numeric.brca.mean)[c(4,6,8)] \# "IL10R_109" "TGFBR1_195" "VEGF_1154"

\#\# Search for the best three-way model

ffit3 <- ssimucross(numeric.brca.mean[1:13],numeric.brca.mean\$time,

numeric.brca.mean\$event, 10,3)

ffit3

table(ffit3\$index)

\#\# 124060740608408117121381213

$\begin{array}{lllllll}\# \# & 1 & 4 & 2 & 1 & 1 & 1\end{array}$

colnames(numeric.brca.mean) $[\mathrm{c}(8,12,13)]$

\section{$R$ code for RSF method}

\#\# VIMP variable selection, get VIMP scores

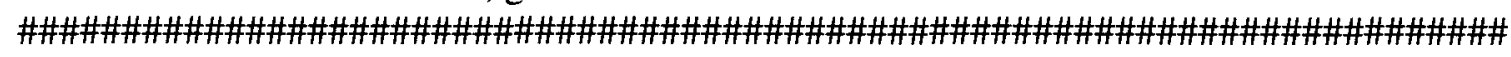

\#\# Fit RSF using 50 different seed values

\#\# Use for loop, change seed each time, keep VIMP scores

library("randomSurvivalForest")

ntree $<-1000$

set.seed(101)

\#\# use log-rank splitting rule, which is the default splitting rule

\#\# use data-imputting (redo 50 repeats using na.impute (but not for poster)

brca.out<- rsf(Survrsf(time, event) ., brca,ntree=ntree, na.action="na.impute")

brca.out\$importance

names(brca)

sort(brca.out\$importance, decreasing=TRUE)

\#\# VIMP KM curve based on one-way model

brca.out\$mortality

q50.mort <- median(brca.out\$mortality)

split.rsf<- ifelse(brca.out\$mortality> q50.mort, 1,0)

library(survival) 
fit.km <- survfit(Surv(brca\$time, brca\$event) split.rsf)

plot(fit.km, main="VIMP KM curve based on one-way model", xlab = "Months from Diagnosis", ylab="Survival Probability", 1ty $=1: 2$, cex $=0.5$ )

legend("bottomright", c("Low risk group", "High risk group"), lty=1:2)

$\mathrm{B}<-50$

VIMP $<-$ matrix (NA, nrow $=13$, ncol $=\mathrm{B})$

rownames(VIMP) <- names(brca)[1:13]

for $(\mathrm{i}$ in $1: \mathrm{B})\{$

brca.multi<- rsf(Survrsf(time, event) .,brca,ntree=ntree, seed $=(100+i)$,na. action $=$ "na.impute" $)$

VIMP[,i] <- brca.multi\$importance

)

VIMP.mean<- rowMeans(VIMP)

VIMP.mean

round(sort(VIMP.mean, decreasing=TRUE),3)

rank.VIMP<- apply (-1*VIMP, 2, rank)

rank.VIMP.mean<- rowMeans(rank.VIMP)

sort(rank.VIMP.mean)

\#\# Minimal Depth variable selection

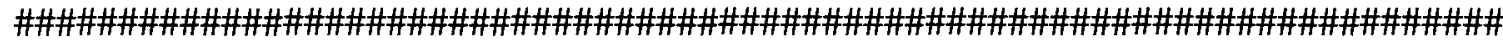

library("randomSurvivalForest")

ntree $<-1000$

nodesize $<-2$

mtry<- $14 *(3 / 4)$

nsplit $<-10$

brca.out<- $\operatorname{rsf}($ Survrsf(time, event) , , brca, ntree $=$ ntree, nodesize $=$ nodesize, mtry=mtry,

"na.impute")

nsplit=nsplit, seed= 101,forest= TRUE,na.action=

sort(brca.out\$importance, decreasing=TRUE)

\#\# Minimal Depth one-way Plot

brca.out\$mortality

q50.mort <- median(brca.out\$mortality)

split.rsf<- ifelse(brca.out \$mortality> q50.mort, 1,0)

library(survival) 
fit.km <- survfit(Surv(brca\$time, brca\$event) split.rsf)

plot(fit.km, main="Minimal Depth KM curve based on one-way model", xlab = "Months from Diagnosis", ylab="Survival Probability", lty=1:2, cex=0.5)

legend("bottomright", c("Low risk group", "High risk group"), lty=1:2)

\#\# Minimal Depth two-way Plot

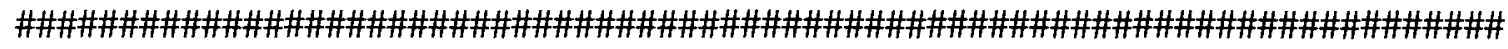

\#\#\#

brca.out<- rsf(Survrsf(time, event) ., brca, ntree=ntree, nodesize=nodesize, mtry=mtry,

"na.impute") nsplit $=$ nsplit, seed $=101$, forest $=$ TRUE, na.action $=$

max.out $2<-$ max.subtree(brca.out, max.order $=2$, sub.order $=$ TRUE)

dist.mat<- as.dist(max.out $2 \$$ subOrder)

clust.snps<- hclust(dist.mat)

plot(clust.snps, xlab = "Months from Diagnosis", cex=1)

B $<-50$

Minimal $<-$ matrix $(\mathrm{NA}$, nrow $=13$, ncol $=\mathrm{B})$

Minimal.mean $<-$ matrix $(\mathrm{NA}$, nrow $=13, \mathrm{ncol}=1)$

rownames(Minimal) <- names(brca) $[1: 13]$

for ( $\mathrm{i}$ in $1: \mathrm{B})$ \{

brca.multi<- rsf(Survrsf(time, event) .,brca,ntree=ntree, nodesize=nodesize, mtry=mtry,

nsplit=nsplit,seed $=(100+i)$, forest= TRUE,na.action= "na.impute" $)$

Minimal[,i] <- brca.multi\$importance

\}

Minimal.mean<-rowMeans(Minimal)

Minimal.mean

round(sort(Minimal.mean, decreasing=TRUE),3)

Minimal.rank<- apply(-1*Minimal, 2, rank)

Minimal.rank.mean<- rowMeans(Minimal.rank)

sort(Minimal.rank.mean) 


\section{CURRICULUM VITAE}

Jie Zhang

E-mail: j0zhan25@louisville.edu

Address: 1345 S $3^{\text {rd }}$ Street Apt\# 4, Louisville, KY, 40208

EDUCATION

2009.08 - Current MS (candidate, expected in May 2011),

Biostatistics, GPA 3.844

University of Louisville (UofL), Louisville, Kentucky, USA

2007.09-2009.06 MS program in Pharmaceutics

China Pharmaceutical University (CPU), Nanjing, China

2003.09 - 2007.06 BE, Bioengineering

China Pharmaceutical University (CPU), Nanjing, China

RESEARCH EXPERIENCE

2010.08--- Now Graduate Student (Guy N. Brock)

Graduate Thesis-Simulation study comparing methods for detecting gene-gene interactions for survival data.

Dept of Bioinformatics and Biostatistics, School of Public Health and Information Sciences, UofL

2010.09---Now Data Analyst \& Lab Assistant (LaCreis R Kidd)

'Innate immunity-related sequence variants as predictors of prostate cancer risk using Multifactor Dimensionality Reduction Method,

Dept of Pharmacology and Toxicology, School of Medicine, UofL

2010.05---2010.09 Data Analyst \& Lab Assistant (Mary Eng)

'Employment outcomes of patients following successful renal transplantation'

Jewish Hospital Transplant Center \&Uof L

2009.10-2010.08 Data Analyst \& Lab Assistant

(G. Rafael Fernandez-Botran)

'IL-4 and IL-4- $\delta 2$ in the modulation of Th1 response to

Mycobacterium tuberculosis among HIV-infected women'

Dept of Pathology and Laboratory Medicine,

School of Medicine, UofL

2009.08-2009.10 Data Analyst \& Lab Assistant (Eugenia Wang)

Bioinformatics Data Analysis 
Gheens Center on Aging,

School of Medicine, UofL

2008.07-2009.06 Graduate Student (JiashengTu)

Worked on project 'Reduction of gelatin cross-linking within

capsules'

Worked as participant of compiling two guide books

$<$ Scientific Design of Experiments -Role in Pharmaceutical

Sciences $>$ and <Guideline of Pharmaceutical Excipients >

Dept of Pharmaceutics, CPU

2007.02 - 2007.06 Bachelor Student (JiashengTu)

Worked on graduation thesis 'The Pharmacokinetics of

Polymeric micelle of Cyclosporine'

Dept of Pharmaceutics, CPU

2005.07-2005.08 Intern quality controller

Nanjing Xiaoying Pharmacy Corp.

PUBLICATIONS

1. Eng M, Zhang J, Cambon A, Brock G, Gleason J. Employment outcomes of Patients following successful renal transplantation. Clinical Transplantation, 2011 Feb 23, accepted

2. Kidd LR, Beache SC, Zhang J, Brock GN, Lillard JW Jr, Rudd J, Kimbro KS. Chemokine and chemokine receptor genetic variants as predictors of aggressive prostate cancer among European-American men. BMC Bioinformatics, submitted

3. Kimbro KS, Beache SC, Zhang J, Lillard JW Jr., Brock GN, Rudd J, Kidd LR. Chemokine and chemokine receptor genetic variants as predictors of breast cancer risk among Caucasian women. BMC Bioinformatics, submitted

4. Fernandez-Botran R, Zhang J, Brock G, Annapan S, Plankey M, Young M, Herold B, Durkin H, Villacres MC, Greenblatt RM, French A, Golub J, Bordon J. Cytokine expression profiles in HIV-infected women preceding tuberculosis. Cytokines in infectious diseases autoimmune and cancer meeting, Chicago, 2010

5. Fernandez-Botran R, Zhang J, Brock G, Annapan S, Plankey M, Young M, Herold B, Durkin H, Villacres MC, Greenblatt RM, French A, Golub J, Bordon J. Cytokine expression profiles in HIV-infected women preceding tuberculosis. Poster, Research! Louisville Poster Competition, Louisville, Oct 11-15, 2010

6. Kimbro KS, Beache SC, Zhang J, Lillard JW Jr., Brock GN, Rudd J, Kidd LR. Chemokine and chemokine receptor genetic variants as predictors of breast cancer risk among Caucasian women. AACR(American Association for Cancer Research) 102nd Annual Meeting, Orlando, FL, April 2-6, 2011

7. Zhang J, Kidd LR, Brock GN, Comparison of Approaches for Detecting Gene-Gene Interactions for Survival Data. Poster, $10^{\text {th }}$ Annual UT-ORNL-KBRIN Bioinformatics Summit, Memphis, TN, April 1-3, 2011

8. Zhang J, Kidd LR, Brock GN, Comparison of Approaches for Detecting Gene-Gene Interactions for Survival Data. Poster, $10^{\text {th }}$ Annual UT-ORNL-KBRIN Bioinformatics Summit, Memphis, TN, April 1-3, 2011 
9. Lavender NA, Rudd J, Zhang J, Hu T, Brock GN, Kimbro KS, Moore JH, Hein DW, Kidd LR. Interaction among apoptosis-associated sequence variants and joint effects on aggressive prostate cancer. Molecular Systems Biology, April, submitted. 